\title{
Terbium(III) phthalocyaninato multiple-decker complexes in high oxidation states: correlation between electronic, structural and magnetic properties and unexpected triplet biradical states
}

Yoji Horii ${ }^{a,}, b *$ Marko Damjanović, ${ }^{c, d *}$ M. R. Ajayakumar, ${ }^{e}$ Keiichi Katoh, ${ }^{a *}$ Yasutaka Kitagawa, Liviu Chibotaru, ${ }^{g}$ Liviu Ungur, ${ }^{h}$ Marta Mas-Torrent, ${ }^{e}$ Wolfgang Wernsdorfer, ${ }^{d}$ Markus Enders, ${ }^{c *}$ Jaume Veciana $^{e *}$ and Masahiro Yamashita ${ }^{a, i, j *}$

${ }^{a}$ Department of Chemistry Graduate School of Science Tohoku University,6-3 Aramaki-Aza-Aoba Aoba-ku, Sendai,Miyagi 980-8578, Japan

${ }^{b}$ Research Center for Thermal and Entropic Science, Graduate School of Science, Osaka University, 1-1 Machikaneyama-cho, Toyonaka, Osaka 560-0043, Japan

${ }^{c}$ Institute of Inorganic Chemistry Heidelberg University Im Neuenheimer Feld 270, 69120 Heidelberg, Germany

${ }^{d}$ Institute of Nanotechnology (INT), Karlsruhe Institute of Technology (KIT), Hermann-vonHelmholtz-Platz 1, 76344 Eggenstein-Leopoldshafen, Germany

${ }^{e}$ Department of Molecular Nanoscience and Organic Materials, Institut de Ciencia de Materials de Barcelona (CSIC)/CIBER-BBN, 08193 Bellaterra, Spain

${ }^{f}$ Department of Materials Engineering Science, Graduate School of Engineering Science, Osaka University, 1-1 Machikaneyama-cho, Toyonaka, Osaka 560-8531, Japan

'Theory of Nanomaterials Group, Katholieke, Universiteit Leuven, 3001 Leuven, Belgium

${ }^{h}$ Department of Chemistry, National University of Singapore, 3 Science Drive 3, 117543 Singapore

${ }^{i}$ School of Materials Science and Engineering, Nankai University, Tianjin 300350, China

${ }^{j}$ WPI-Advanced Institute for Materials Research, Tohoku University, 2-1-1 Katahira, Sendai 980-8577, Japan

\begin{abstract}
In this article, we present a comprehensive study of the highly oxidized species of multiple-decker complexes composed of Terbium and Cadmium ions and two to five phthalocyaninato ligands, which are stabilized by electron-donating $n$-butoxy chains. Highly extended $\pi$-radical delocalization enables us to isolate up to $+3 e$ charged states for triple-decker complexes, $+4 e$ charged states for quadrupledecker complexes and $+5 e$ charged states for quintuple-decker complexes. Paramagnetic ${ }^{1} \mathrm{H}$ NMR measurements for the series of triple, quadruple and quintuple-decker complexes revealed that ligand oxidations lead to a decrease in magnetic anisotropy. These observations, along with the results from magnetometry measurements, are in good agreement with predictions from ab initio calculations. In addition, unusual paramagnetic shifts were observed in $+2 e$ charged quadruple and quintuple-decker complexes, indicating that those two species are actually triplet biradicals, contrary to expectations.
\end{abstract}


With the combination of the ${ }^{1} \mathrm{H}$ NMR analysis and DFT calculations, we revealed that the radicals on $+2 e$ charged states are localized on the edges of the multiple-decker units. The X-ray structural analysis for series of oxidized multiple-decker complexes revealed that all the species show an axial compression induced by the ligand oxidations, resulting in the bowl-shaped distortion of the ligands, which agrees with predictions from DFT calculations. In $+4 e$ charged quadruple- and quintuple-decker complexes, the unusual coexistence of square antiprism (SAP) and square prism (SP) coordination geometries around $\mathrm{Tb}(\mathrm{III})$ ions supported by solvent incorporation in one multiple-decker unit was observed. Magnetic measurements revealed that the series of these deckers shows single-molecule magnet (SMM) properties, which are controlled by the multi-step redox induced structural changes.

\section{INTRODUCTION}

Construction of extended $\pi$-systems is one of the main topics in synthetic chemistry. ${ }^{1-2}$ The versatile physical properties of these, such as redox, light absorption and emission and electroconductivity closely relate to the applications in solar cells, ${ }^{3}$ organic light emitting diodes, ${ }^{4}$ electro-batteries ${ }^{5-6}$ and so on. There are two kinds of $\pi$-extension, one of which is in-plane or lateral extension, and the other being the longitudinal extension, so called $\pi-\pi$ stacking. ${ }^{7}$ The bottom up synthesis of the graphene nano-ribbon ${ }^{2,8}$ and the construction of porphyrin tape ${ }^{9}$ are representative examples of the in-plane extension of $\pi$-system, in which the precisely designed monomer units are connected regularly with $\mathrm{sp}^{2}$ bonds, making it possible to control the morphology and the physical properties of resulting products. Longitudinal infinite stacking of $\pi$-conjugated molecules is often seen in molecular conductors in crystals ${ }^{10-13}$ and polymeric chains in which the phthalocyanine $(\mathrm{Pc})$ and/or porphyrin (Por) are connected by siloxane, ${ }^{14}$ ligand-metal coordination bond, ${ }^{15}$ weak intermolecular interactions ${ }^{16}$ and so on. In addition to the infinite stacks, the development of the Pc and Por chemistry enables one to construct the stable oligomeric molecules with stacked $\pi$-system. ${ }^{15,17-19}$ The series of phthalocyaninato-lanthanide-cadmium multiple-decker complexes ${ }^{20-22}$ are one rare example of such oligomers. The history began with the first report of lanthanoid-phthalocyaninato double- and tripledecker complexes as achieved by Kirin et al. in $1965^{23}$ and in $1967^{24-25}$, followed by the synthesis of their porphyrinato analogues reported and Buchler et al. ${ }^{26}$ Use of the mono nuclear lanthanides Pc and Por complexes, namely single-decker complexes, as the precursor allows the selective synthesis for mixed Pc-Por double- and hetero metallic triple-decker complexes. ${ }^{27-28}$ Following the improvements in synthetic chemistry, the detailed analyses for electronic, ${ }^{29-37}$ structural ${ }^{38-39}$ and magnetic properties $^{34-37,40-41}$ of these complexes were done, revealing that these can be utilized for sensors, ${ }^{42}$ transistors, ${ }^{43-44}$ molecular rotors ${ }^{45-47}$ and excellent single-molecule magnets. ${ }^{40}$ After a long while since these studies, the first discrete quadruple-decker complex has been reported by Fukuda et al in 2011, in which two $\mathrm{Lu}(\mathrm{III})-\mathrm{Pc}$ double-decker complexes are connected by $\mathrm{Cd}(\mathrm{II})$, resulting in the tetrameric Pc stack connected by Lu-Cd-Lu metal array. ${ }^{21}$ This novel strategy was further extended by Jiang et 
$a l$, and the quintuple- and sextuple-decker complexes have been reported in a short period of time. ${ }^{21-}$ ${ }^{22}$ Among the multiple-decker complexes, double-decker complexes are the most thoroughly studied, $34,40-41,48$ one possible reason being their versatile functionalities related to future applications. For instance, redox induced structural changes of Por double-decker complexes, which are expected theoretically and indirectly proofed experimentally, are utilized for rotating units of various molecular machines. ${ }^{45-47}$ Very recently, Yamashita et al. have reported structural changes induced by ligand oxidations in Por double-decker complexes using single-crystal X-ray (SXRD) measurements and revealed that the ligand oxidations induce the axial compression, as expected by empirical and DFT calculations. ${ }^{49}$ The axial compression in Pc double-decker complexes induced by oxidation is also investigated by solution NMR studies ${ }^{48}$. The conformational changes, especially the ligand rotations in double-decker complexes have been directly observed ${ }^{34-37,40-41,50}$ and controlled using scanning electron microscope (STM) and scanning electron spectroscopy (STS), thus the single molecule memory using double-decker complexes has been achieved. ${ }^{43,51}$ In addition to the structural properties, double-decker complexes with $\mathrm{Tb}(\mathrm{III})$ ions are outstanding $\mathrm{SMMs}^{44,}$, 52-54 and used for spintronic devices, utilizing the interactions between spins of $\mathrm{Tb}(\mathrm{III})$, conductive electrons and phonons of nanocarbon materials. ${ }^{55-59}$ Compared with double-decker complexes, the redox, structural and magnetic properties of other multiple-decker complexes are much less investigated, presumably being due to the short history and the low-profile, difficulty in synthesis, purification and low crystallinity. However, the more extended $\pi$-systems compared to double-decker complexes are promising for the valuable and unprecedented functionalities. For instance, Fukuda et al. have reported that the monooxidized form of quadruple-decker complexes shows light absorption in the longer wavelength regions. ${ }^{60}$ Light absorption in the wide range of wavelengths is helpful for light-harvesting materials in dye sensitized solar cells, for gathering the light from the sun as much as possible. In addition, nonlinear optical properties of multiple-decker complexes have been investigated by Jiang et al. ${ }^{22}$ From the point of view of molecular magnetism, various groups utilize the multiple-decker complexes having two $\mathrm{Tb}(\mathrm{III})$ and $\mathrm{Dy}(\mathrm{III})$ centers for analyzing the effect of intramolecular magnetic interactions which should affect the SMM properties. ${ }^{44,52-54}$

In this article, we present a comprehensive study of oxidized multiple-decker complexes obtained through electrochemical and chemical oxidations, utilizing theoretical calculations, paramagnetic NMR measurements, single crystal X-ray diffraction (SXRD) and magnetic measurements. These were done in order to gain a deeper understanding for physical properties of longitudinally extended $\pi$-systems which may lead to various functionalities. Because the multiple-decker complexes in this research are composed from the 2,3,9,10,16,17,23,24-octabutoxy phthalocyaninato (obPc) ligands having electron donating $n$-butoxy chains, the oxidized states of them are stabilized. By using the bulk electrolysis and UV-Vis-NIR measurements, we isolated and characterized the charged states of multiple-decker complexes. To avoid confusion in the naming, we use here the numbers of obPc 
ligands (n) contained in the complex, and the name of them is defined as [n], i.e. double- [2], triple [3], quadruple- [4] and quintuple-decker [5] complexes. The charge of the complex (m) is expressed as the superscript as $[\mathbf{n}]^{\mathbf{m}+}$, e.g. $+2 e$ charged triple-decker complex is named $[3]^{2+}$. We characterized the structures and magnetic properties in solution using paramagnetic NMR analysis in the oxidized complexes and revealed that magnetic anisotropy decreases with increasing the charges on the obPc ligands, in agreement with magnetic measurements and theoretical predictions. Furthermore, even though the $[4]^{2+}$ and $[5]^{2+}$ have even numbers of positive charges, the existence of $\pi$-radicals was proven by NMR, ESR and supported by DFT calculations. Among multiple-decker complexes, we succeeded in getting the crystal structures of $[2]^{-},[2]^{+},[3]^{2+},[4]^{2+},[4]^{4+}$ and $[5]^{4+}$ and revealed that both molecular and packing structures of them are controlled by the size of the molecules and numbers of counter ions. SMM properties of the multiple-decker complexes alter by the structural effect induced by chemical oxidations and are also reported in this work.

\section{RESULTS AND DISCUSSIONS}

\section{Electrochemical analysis of multiple-decker complexes}

Redox behavior of the multiple-decker complexes has been briefly reported in our previous article. ${ }^{61}$ Figure 1 summarizes the multi-redox wave of cyclic voltammograms and redox potentials of the multiple-decker complexes in $\mathrm{CH}_{2} \mathrm{Cl}_{2}$ containing $100 \mathrm{mM}$ of tetrabutylammonium hexafluorophosphate $\left(\mathrm{TBA} \cdot \mathrm{PF}_{6}\right)$ and $0.5 \mathrm{mM}$ of charge neutral complexes. The numbers of redox waves increase with increasing the numbers of obPcs due to the longitudinal extension of the $\pi$ systems. Since $\mathrm{Tb}(\mathrm{III})$ and $\mathrm{Cd}(\mathrm{II})$ do not participate in the redox reactions, these redox waves purely originate from the extended $\pi$-system composed of obPcs. In case of [2], two reduction and two oxidation waves were observed. [3] shows three reduction and four oxidation waves in CV. However additional reduction waves at $-2.5 \mathrm{~V} v \mathrm{vs}$. $\mathrm{Fc} / \mathrm{Fc}^{+}$were observed in DPV measurements. The number of redox waves in [4] is the same as observed for [3]. However, the oxidation waves and the reduction waves shift toward lower and higher potential regions, respectively. [5] shows four reduction and five oxidation waves in the measurement range. Redox potentials of the multiple-decker complexes are summarized in Figure 1. Potential differences of each of the redox waves become narrower with increasing the number of stacks because the extension of $\pi$-system decreases the electronic repulsion between surplus electrons in reduced species or holes in oxidized species. In those series, there is an anomaly of redox behavior in [2] because the neutral form of [2] ([2] $\left.]^{0}\right)$ owns a $\pi$-radical, which is different from other neutral complexes having no $\pi$-radicals. Comparison of [3], [4] and [5] indicates that first reduction and first oxidation potentials, which are attributed to the LUMO and HOMO levels, show an increase with increasing the stacking number. Generally, extension of the $\pi$-system increases HOMO levels and decreases LUMO levels, giving rise to the smaller HOMO-LUMO gaps. The negative shift of first reduction potential with increasing the number of ligands has been observed in 
silicon phthalocyaninato oligomers as well, ${ }^{19}$ indicating that redox potentials are not determined solely by charge delocalization in the extended $\pi$-system. To investigate electronic structure in the present complexes, DFT geometry optimizations at the (U)TPSSH/Def2-SVP level ${ }^{62-64}$ were performed. To reduce the computational cost, $\mathrm{Tb}$ (III) ions are changed to diamagnetic $\mathrm{Y}$ (III) ions of which chemical properties and molecular structure are similar (or almost identical) to those of $\mathrm{Tb}(\mathrm{III})$, and the $n$-butoxy chains are changed to methoxy ones. HOMO and LUMO of [3], [4] and [5] are summarized in Figure 1c. The electrons in HOMOs extend over the molecules, whereas the electrons of LUMOs tend to localize on the inner ligands. From the point of view of localized orbitals scheme, the ligands in the multiple-decker complexes have higher energies than free ligands because the ligands in the former situation feel the electronic repulsion by the adjacent ligands. The numbers of the adjacent ligands of outer and inner ligands are one and two respectively, making the inner ligands less stable. In addition, difference in the charge of $\mathrm{Tb}(\mathrm{III})$ and $\mathrm{Cd}(\mathrm{II})$ should affect the electron-withdrawing properties of coordinating obPc. With increasing the number of obPc, the numbers of less charged $\mathrm{Cd}$ (II) shared with inner obPcs increase, resulting in the less electron-withdrawing nature of inner obPc which increases the LUMO levels. According to these assumptions, the LUMO level of the [5] will increase as seen in the electrochemical measurements because of the localized electrons on three inner ligands. The theoretical calculations in gas phase predict that LUMO level in $[\mathbf{5}]^{0}$ becomes lowest among the studied complexes. Inclusion of solvation effects using the polarizable continuum model (PCM $)^{65}$ makes the LUMO levels of $[3]^{0}$ lowest and those of $[4]^{0}$ and $[5]^{0}$ close to each other in energy. Therefore, the theoretical predictions with PCM included are closer to experimental results. However, the shifts of HOMO and LUMO levels among neutral multiple-decker complexes $(\sim 0.05 \mathrm{eV})$ are small compared to the shift of first oxidation and reduction potentials $(\sim 0.1 \mathrm{eV})$, indicating the importance of counter ions for determining the redox potentials. The smaller the molecular size is, the more the ionic states are stabilized by the counter ions. Thus, we suspect that LUMO electrons localized in inner ligands as well as the solvation by counter ions rise the first reduction potentials with increasing the number of ligands. 
(a)

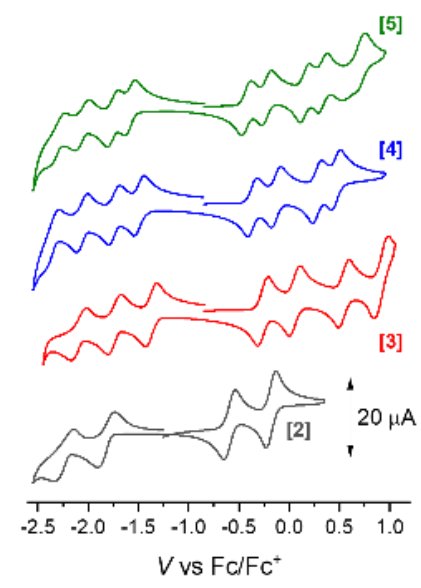

(b)

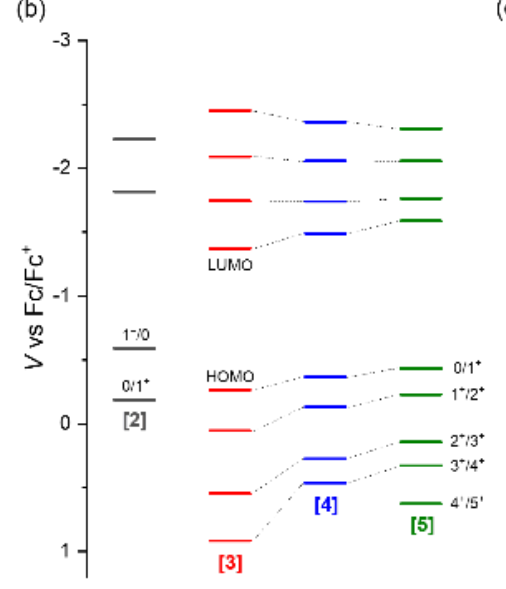

(c)

HOMO

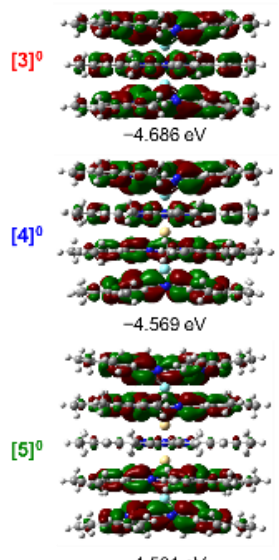

$-4.521 \mathrm{eV}$
LUMO

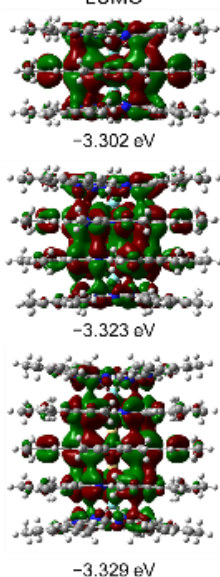

Figure 1. (a) Cyclic voltammograms and (b) redox potentials for multiple-decker complexes at room temperature. Both electrochemical data were referred from our previous article. ${ }^{61}$ Each redox waves in oxidized regions were characterized using electrochemical UV-Vis-NIR absorption spectra. (c) Electron map and energy levels of LUMO and HOMO of [3] ${ }^{0},[4]^{0}$ and $[5]^{0}$ with PCM model (isovalue $=0.01$ ). The electrons in LUMO localize on inner ligands, making the LUMO levels higher with increasing the numbers of stacks.

Redox properties of the multiple-decker complexes were further investigated using in-situ UV-VisNIR spectroscopy during the bulk electrolysis in $\mathrm{CH}_{2} \mathrm{Cl}_{2}$ containing $0.01 \mathrm{mM}$ of samples and $100 \mathrm{mM}$ of $\mathrm{TBA} \cdot \mathrm{PF}_{6}$. To prevent the evaporation of the solvent, the cell for electrolysis was sealed with a rubber septum. Figure 2 shows the changes in UV-Vis-NIR spectra in $\mathrm{CH}_{2} \mathrm{Cl}_{2}$ during the electrolysis. The completion of the electrochemical reactions was confirmed by the decrease of current down to $\sim 1 \%$ of the initial value. The present multiple-decker complexes have $n$-butoxy chains at peripheral $(\beta)$ positions of phthalocyaninato ligands. The electron donating nature of $n$-butoxy chains destabilizes reduced states and stabilizes oxidized states. This was confirmed by the fact that the reduced states of the multiple-decker complexes go back to neutral states immediately in the air whereas those of oxidized states are stable for a while in the air. ${ }^{48}$ Figure 2 shows transient absorption spectra during electrochemical oxidations. All spectra shown here give isosbestic points, indicating no decomposition during the oxidation reactions. The numbers of the possible oxidized species correlate with the number of the phthalocyaninato ligands. In case of the [3], up to $+3 e$ charged states were isolated using bulk electrolysis. Oxidation from $[3]^{3+}$ to $[3]^{4+}$ gave no isosbestic points, indicating that $[3]^{4+}$ is unstable under the given experimental conditions. [4] series shows stable oxidized species up to the $+4 e$ charged state. In case of [5] series, isosbestic points between $[5]^{4+}$ and $[5]^{5+}$ states were observed. In other words, the number of the stable oxidized states increased with increasing the numbers of the stacks. Longitudinal extension of the $\pi$-system enabled the delocalization of positive charges of oxidized 
species and stabilized the highly oxidized states effectively.

The changes in absorption spectra of [2] are almost identical to those found in the non-substituted lutetium(III) double-decker complexes extensively studied by Ishikawa et al. ${ }^{30-31,33} \mathrm{~A}$ sharp and strong absorption at $15840 \mathrm{~cm}^{-1}$ and smaller peaks at $14740 \mathrm{~cm}^{-1}$ in [2] are HOMO to degenerated LUMO+1 orbitals and HOMO-1 to degenerated LUMO respectively. The weak absorption at $6330 \mathrm{~cm}^{-1}$ in [2] is related to the $\pi$-radical on the ligand, which has been observed at $\sim 7100 \mathrm{~cm}^{-1}$ in unsubstituted complexes, ${ }^{66}$ and it corresponds to the transition from the highest two-electron filled molecular orbital to a singly occupied molecular orbital (|LUMO $\beta \leftarrow H O M O \beta>$ transition). This band shifts to 10000 $\mathrm{cm}^{-1}$ in $[2]^{+}$, which corresponds to a HOMO-LUMO transition. In contrast to $[2]^{\mathbf{0}}$, colors of $[3]^{\mathbf{0}}$ and $[4]^{0}$ are visibly distinguishable from those of unsubstituted ones because of the bathochromic shift and broadening of a strong absorption around $16500 \mathrm{~cm}^{-1}(600 \mathrm{~nm})$ induced by $n$-buoxy chains. TD-DFT calculations for Y(III) analogues of closed-shell species were conducted in order to characterize the absorption spectra. Two largest oscillator strengths $f$ for $[3]^{0}$ are obtained at $15376 \mathrm{~cm}^{-1}$ and 15377 $\mathrm{cm}^{-1}$ (both are $f=0.6068$ ), being consistent with the experimental spectra showing the strong absorption around $15600 \mathrm{~cm}^{-1}$. Both exited states contain a lot of configurations, but mainly stem from $\mid \mathrm{LUMO}+2 \leftarrow \mathrm{HOMO}-1>$ and $\mid \mathrm{LUMO}+3 \leftarrow \mathrm{HOMO}-1>$, respectively. The second largest oscillator strengths are given around $19000 \mathrm{~cm}^{-1}(f=0.176-0.1717)$, where four excited states composed of a lot of configurations are predicted. The transitions to these excited states cause the electron transfer among the inner obPc (Figure S285). The characteristic absorption of $[3]^{2+}$ at $6250 \mathrm{~cm}^{-1}$ was reproduced by TD-DFT calculations, which corresponds to $\mid \mathrm{LUMO} \leftarrow \mathrm{HOMO}>\operatorname{transition}\left(5604 \mathrm{~cm}^{-1}\right.$, $f=0.3751$ ) accompanying the electron transfer from the outer to the inner obPc. The oscillator strengths at $14400 \mathrm{~cm}^{-1}$ and $16100 \mathrm{~cm}^{-1}$ correspond to the absorption at $14100 \mathrm{~cm}^{-1}$ and the shoulder peak at $15700 \mathrm{~cm}^{-1}$ in experimental spectra, respectively. These transitions induce the electron transfer from the phenyl rings obPc to the pyrrole ring in obPc ligands. In $[4]^{0}$, the strong absorption at 15300 $\mathrm{cm}^{-1}$ with a shoulder at $16900 \mathrm{~cm}^{-1}$ was observed, although the single absorption peak has been observed in unsubstituted and tert-butyl substituted analogues. Two distinct $f$ at $15424 \mathrm{~cm}^{-1}(f=$ $0.1939)$ and $16789 \mathrm{~cm}^{-1}(f=0.3432)$ are consistent with the experimental spectra. In addition, the broad tail extended over $\sim 7000 \mathrm{~cm}^{-1}$ was observed, at which the $\mid$ LUMO $\leftarrow$ HOMO $>\left(8708 \mathrm{~cm}^{-1}, f\right.$ $=0.0095)$ is predicted. In the case of $[4]^{2+}$, the characteristic broad absorption at $5880 \mathrm{~cm}^{-1}$ is attributed to $\mid \mathrm{LUMO} \leftarrow \mathrm{HOMO}-1>$ transition with the electron transfer from inner to outer obPcs. A $\mid$ LUMO $\leftarrow$ HOMO $>$ transition at $1433 \mathrm{~cm}^{-1}(f=0.0450)$ in $[4]^{2+}$ is predicted by TD-DFT calculations, which is out of range of the absorption measurements and longer than that of mono-oxidized state of tert-butoxy substituted quadruple-decker complexes reported by Fukuda et al. $\left(3065 \mathrm{~cm}^{-1}\right) \cdot{ }^{67}$ Comparison of solid state IR spectra between the [4] series reveals no additional peaks in $[4]^{2+}$ at predicted range of $\mid \mathrm{HOMO}$ $\leftarrow$ LUMO $>$ absorption because of the overlapped vibrational absorption (Figure S336). In $[4]^{4+}$, the absorption at $7900 \mathrm{~cm}^{-1}$ is attributed to the $\mid \mathrm{HOMO}+1 \leftarrow$ LUMO- $1>$. Although a $\mid \mathrm{HOMO} \leftarrow$ LUMO $>$ 
transition is predicted at $6248 \mathrm{~cm}^{-1}(f=0.0632)$, no obvious absorptions were experimentally observed because of the small $f$ value. As will be discussed later, the HOMO-LUMO energy gap of $[4]^{2+}$ is extraordinarily smaller than that of other [4] species, thus lowering energy of $\mid \mathrm{HOMO} \leftarrow$ LUMO $>$ transition. Although the observation of this transition is impossible in our experimental setup, the lower HOMO-LUMO gap is expected experimentally due the fact that only $[4]^{2+}$ shows a biradical character among [4] series, according to the NMR and ESR analyses shown in the next section. In addition, it is worth noting that the $+2 e$ charged state of a quadruple-decker complex substituted with 16 tert-butyl groups reported by Fukuda et al. is ESR silent, indicating the importance of the 32 electron donating $n$-butoxy groups for lowering the HOMO-LUMO gaps. Although TD-DFT calculations for [5] did not show convergence due to huge molecular structure, the spectra of them are resemble to those of [4]. In addition, $[5]^{2+}$ shows biradical character as seen in $[4]^{2+}$ as discussed in NMR part, proofing the lower HOMO-LUMO gap than other charged states.

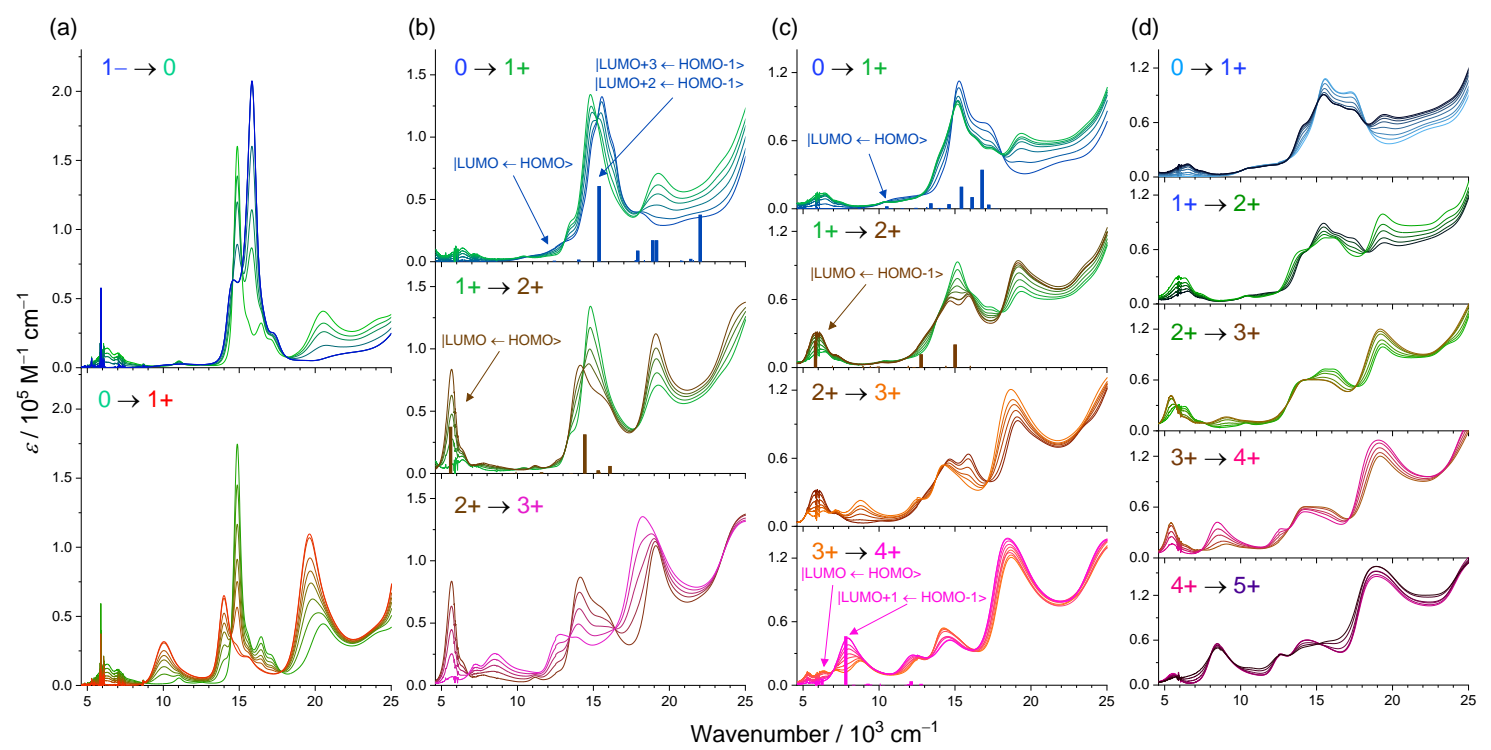

Figure 2. Electrochemical UV-Vis-NIR spectra for (a) [2], (b) [3], (c) [4], (d) [5] series. The spectra giving the isosbestic points and the oscillator strength are shown.

\section{Solution NMR measurements}

Paramagnetic solution NMR is a powerful tool to unveil the molecular structure and the magnetic properties of lanthanoid-based complexes because the magnitude of the paramagnetic shift is affected by the positions of the measured nuclei relative to spin density, as well as by the magnetic anisotropy of lanthanide ions. ${ }^{68-69}$ The chemical shift of an NMR active nucleus in a paramagnetic complex can be expressed as the sum of a temperature-independent orbital term $\left(\delta_{\text {orb }}\right)$ and temperature-dependent hyperfine term $\left(\delta_{\mathrm{HF}}\right) . \delta_{\mathrm{HF}}$ is mainly composed of the Fermi contact shift $\left(\delta_{\mathrm{FC}}\right)$ and pseudocontact shift $\left(\delta_{\mathrm{PC}}\right)$ as shown in Eq. $1{ }^{48,70-77}$

$$
\delta_{\mathrm{obs}}=\delta_{\mathrm{orb}}+\delta_{\mathrm{HF}}=\delta_{\mathrm{orb}}+\delta_{\mathrm{FC}}+\delta_{\mathrm{PC}}
$$


$\delta_{\mathrm{FC}}$ stems from the interactions between the electron spin on the nucleus and the nuclear spin. In other words, $\delta_{\mathrm{FC}}$ depends on the spin densities on the measured nuclei. $\delta_{\mathrm{PC}}$ originates from the throughspace magnetic dipole-dipole interactions between the electron magnetic moment and the nuclear magnetic moment. The contribution of the $\delta_{\mathrm{PC}}$ becomes prominent when the metal complexes possess strong magnetic anisotropy. ${ }^{78}$ Both the magnetic metal ions and the ligand-centered $\pi$-radicals contribute to the $\delta_{\mathrm{FC}}$ and $\delta_{\mathrm{PC}}$, as is shown in Eq.2

$$
\delta_{\text {obs }}=\delta_{\text {orb }}+\delta_{\mathrm{MFC}}+\delta_{\mathrm{MPC}}+\delta_{\mathrm{LFC}} \quad \text { Eq. } 2
$$

where $\delta_{\mathrm{MPC}}$ and $\delta_{\mathrm{MFC}}$ represent the Fermi contact term and pseudocontact term from magnetic metal ions and $\delta_{\mathrm{LFC}}$ represents the contribution from the ligand $\pi$-radical. The pseudocontact shift of the ligand $\pi$-radicals $\left(\delta_{\mathrm{LFC}}\right)$ is normally omitted because of rather small contributions due to the insignificant magnetic anisotropy of organic radicals. ${ }^{79}$ Needless to say, $\delta_{\mathrm{LFC}}$ vanishes when the metal complexes have no $\pi$-radicals on the ligands ${ }^{48}$ Localized f-orbitals, which play a decisive role in the magnetism of lanthanoid ions, enable us to treat the $\delta_{\mathrm{MPC}}$ using a point-dipole approximation. The $\mathrm{Tb}$ (III) ions in the present complexes are affected by the ligand field of obPc ligands, thereby the $\mathrm{Tb}(\mathrm{III})$ ions possess the strong axial magnetic anisotropy where the magnetic easy axis lies along the $\mathrm{C}_{4}$ axis of the multiple-decker complexes. ${ }^{79}$ In this case, the $\delta_{\mathrm{MPC}}$ is expressed ${ }^{68}$ as

$$
\delta_{\mathrm{MPC}}=\frac{\chi_{\mathrm{ax}}}{12 \pi r^{3}}\left(3 \cos ^{2} \theta-1\right) \quad \text { Eq. } 3
$$

where $\chi_{\text {ax }}$ is the axial component of the magnetic susceptibility of the lanthanoid center, $r$ is the norm of the vector $(\boldsymbol{r})$ connecting the lanthanoid center and the nucleus, $\theta$ is the angle between the magnetic easy axis and the $\boldsymbol{r}$. The contribution of the $\delta_{\mathrm{MFC}}$ to the nuclei distant from the lanthanoid center is small compared to $\delta_{\text {MPC }}$ because the overlaps between the f-orbitals and the orbitals of the measured nuclei (H and C) are small. In our previous research, we have reported the NMR spectra of [2] series and revealed that the paramagnetic shift of these complexes can be expressed by Eq. 2 and Eq. 3. ${ }^{48,78}$ As mentioned above, the $\delta_{\text {MPC }}$ shows dominant contributions to the paramagnetic shift of non-radical $[2]^{-}$and $[2]^{+}$, giving rise to large paramagnetic shifts toward negative regions. The overall ${ }^{1} \mathrm{H}$ NMR spectra of $[2]^{-}$and $[2]^{+}$are similar with each other because the structural formulas of the molecular units are unchanged by the chemical reactions. However, $[2]^{+}$shows a larger paramagnetic shift compared to $[2]^{-}$. Finally, we have concluded that decrease of interligand distance $\left(R_{\mathrm{obPc}-\mathrm{obPc}}\right)$ and the increase in $\chi_{\mathrm{ax}}$ value contribute to the larger $\delta_{\mathrm{MPC}}$ induced by the ligand oxidation. ${ }^{48}$ However, in contrast to the [2], the series of complexes from [3] to [5] does not show a simple increase in $\delta_{\mathrm{MPC}}$ by the ligand oxidations, as discussed below.

The oxidized complexes for NMR measurements were prepared by mixing the neutral complexes with the oxidizer (phenoxathiin hexachloroanitimonate) in $\mathrm{CD}_{2} \mathrm{Cl}_{2}$. Oxidation numbers of the complexes were controlled by changing the amount of oxidizer as precisely as possible and confirmed by comparing the UV-Vis spectra of the samples with those obtained by bulk electrolysis. Figure 3 
summarizes the annotations in ${ }^{1} \mathrm{H}$ NMR. As is shown in Figure 3, obPc ligands can be distinguished as either inner (obPc ${ }^{i}$ and, for the central ligand in [5], obPcii) and outer $\left(\mathrm{obPc}^{\circ}\right)$. The ${ }^{1} \mathrm{H}$ NMR signals were fully assigned with the combination of ${ }^{1} \mathrm{H}$ NMR spectra, ${ }^{1} \mathrm{H}-{ }^{1} \mathrm{H}$ COSY spectra and estimation of $\delta_{\text {MPC }}$ using DFT optimized structures. In case of some of the oxidized complexes it was more difficult to observe crosspeaks in the ${ }^{1} \mathrm{H}-{ }^{1} \mathrm{H}$ COSY spectra (broader signals). Therefore, the NMR signals of these complexes were assigned according to the $\delta_{\mathrm{MPC}}$ calculated using Eq. 3 and DFT-optimized structures, shapes of signals (coupling, FWHM) and comparison with the non-radical species. $\delta_{\text {orb }}$ was estimated by ${ }^{1} \mathrm{H}$ NMR signals for Y(III) analogues of multiple-decker complexes at room temperature. As shown in Figure 4, the ${ }^{1} \mathrm{H}$ signals of the $\mathrm{CH}_{\mathrm{ar}}$ show complicated changes induced by the oxidations, where the magnitude of paramagnetic shift shows both increase and decrease due to the oxidations. In contrast, most of the signals of $\mathrm{CH}_{2} \alpha, \mathrm{CH}_{2} \beta, \mathrm{CH}_{2} \gamma$ and $\mathrm{CH}_{3}$ show a decrease in the paramagnetic shift with increasing the oxidation numbers, which is indicative of a decrease in $\chi_{\text {ax. }} . \delta_{\text {obs }}$ of $\mathrm{CH}_{\text {ar }}$ protons heavily depends on the $\delta_{\mathrm{LFC}}$ because these protons are close to the $\pi$-radicals of the ligands. In contrast, ${ }^{1} \mathrm{H}$ NMR analysis for Y(III) analogues of the oxidized multiple-deckers indicate that changes of the $\delta_{\text {orb }}(\sim 1 \mathrm{ppm})$ are sufficiently small compared to those of $\delta_{\mathrm{LFC}}(\sim 10 \mathrm{ppm})$ in order to be ignored. Consequently, the chemical shift of the $\mathrm{CH}_{\mathrm{ar}}$ is simplified as the sum of $\delta_{\mathrm{MPC}}, \delta_{\mathrm{LFC}}$ and less charge dependent $\delta_{\text {orb }}$ as shown below.

$$
\delta_{\mathrm{obs}}^{a r} \approx \delta_{\mathrm{orb}}+\delta_{\mathrm{MPC}}+\delta_{\mathrm{LFC}} \quad \text { Eq. } 4
$$

The $\delta_{\mathrm{LFC}}$ of $\mathrm{CH}_{\mathrm{ar}}$ is estimated by the spin densities at measured nuclei obtained from the DFT calculations. ${ }^{48}$ The spin densities on the $\mathrm{CH}_{\mathrm{ar}}{ }^{\circ}$ tend to be high compared to those of $\mathrm{CH}_{\mathrm{ar}}{ }^{\mathrm{i}}$, thereby the magnitude relation of the $\delta_{\text {obs }}^{a r}$ among $\mathrm{CH}_{\mathrm{ar}}{ }^{\mathrm{o}}$ and $\mathrm{CH}_{\mathrm{ar}}{ }^{\mathrm{i}}$ changes by the oxidations as seen in the ${ }^{1} \mathrm{H}$ NMR spectra of [4] and [5] series beyond $-55 \mathrm{ppm}$.

In contrast to $\mathrm{CH}_{\text {arr }}$, the protons of the butoxy chains (i.e. $\mathrm{CH}_{2} \alpha, \mathrm{CH}_{2} \beta, \mathrm{CH}_{2} \gamma$ and $\mathrm{CH}_{3}$ ) are less affected by the $\pi$-radicals and charges on the ligands because these protons are far from the $\pi$-plane, making it possible to treat the $\delta_{\mathrm{FC}}$ as zero. ${ }^{48}$ In this situation, the chemical shifts of the butoxy protons $\delta_{\mathrm{obs}}^{b u}$ are expressed as the sum of $\delta_{\text {orb }}$ and the geometry-dependent $\delta_{\text {MPC }}$ as shown below.

$$
\delta_{\text {obs }}^{b u} \approx \delta_{\text {orb }}+\delta_{\text {MPC }} \quad \text { Eq. } 5
$$

Using the Eq. 3, Eq. 5 and the DFT-optimized structures of oxidized complexes to which $n$-butoxy chains were added (Figure S254, S262 and S273), the $\chi_{\text {ax }}$ values are determined from fitting as to minimize the difference in the experimental and expected $\delta_{\text {obs }}^{b u}$ using the least squares method. After getting the $\chi_{\mathrm{ax}}$ values, the quality of fitting was evaluated by the agreement factor $(A F)$ taking into account the $\delta_{\text {obs }}^{a r}$, as summarized in Figure 5, showing the consistency between the theoretical and the experimental pseudocontact shift. The $\chi_{\mathrm{ax}}$ values of the multiple-decker complexes are shown in Figure 6. Except for the increase in the $\chi_{\mathrm{ax}}$ value by the oxidations from $[3]^{2+}$ to $[3]^{3+}, \chi_{\mathrm{ax}}$ values of the multiple-decker complexes decrease with increasing the number of charges on the ligands, indicating that the magnetic anisotropy decreases due to changes induced by the chemical oxidations. 
The results of the [3] series are in contrast to the NMR results of the [2] series, where the $\chi_{\text {ax }}$ increases with oxidation. As is discussed in the section of the magnetic analysis, the reduction of $\chi_{\mathrm{ax}}$ of $\mathrm{Tb}$ (III) ions by the oxidations is the inherent nature of multinuclear complexes. The difference between the [2] series and other complexes is in the number of the metal ions inside the molecules. The ligand oxidations lead to an axial compression as well as to a decrease in intramolecular metal-metal distances ( $\mathrm{Tb}-\mathrm{Tb}$ distance for [3] and $\mathrm{Tb}-\mathrm{Cd}$ distances for [4] and [5]), decreasing the axial components of the ligand field, thereby decreasing the magnetic anisotropy. The small $\chi$ ax values in [3] compared to [4] and [5] series comes from the difference between the $+3 e$ charges on $\mathrm{Tb}$ (III) and $+2 e$ charges on $\mathrm{Cd}$ (II) ions adjacent to the $\mathrm{Tb}(\mathrm{III})$ ions, the former making the axial component of negative charges smaller than the latter do. One possible explanation for the discrepancy observed in the $[3]^{2+}$ case (Figure 6) is that, as expected, the longitudinal compression due to oxidation reduces the $\chi_{\text {ax }}$ value. However, in the $[3]^{3+}$ and $[3]^{+}$species the presence of organic radicals on the ligands counters this effect on the $\chi_{\mathrm{ax}}$ value. This shielding effect from the organic radicals is less pronounced for the larger deckers, where $\pi$ radicals are mostly located on the peripheral ligands, and very little between the $\mathrm{Tb}$ (III) ions (please see spin density distributions in Figure S257 and S260).

(a)

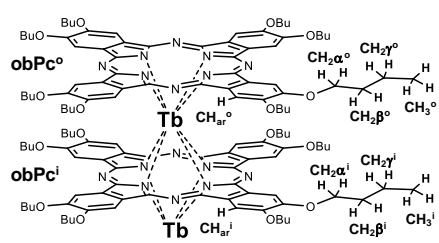

(b)

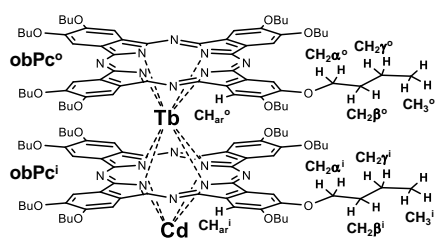

(c)

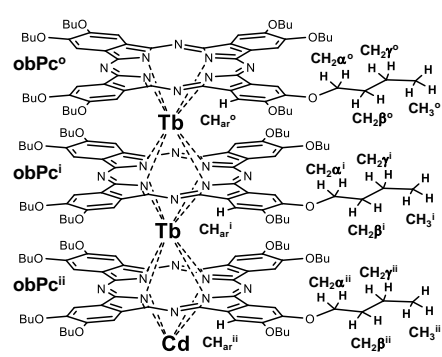

Figure 3. Schematic structures of (a) [3], (b) [4] and (c) [5] with annotations of ${ }^{1} \mathrm{H}$ observed in the ${ }^{1} \mathrm{H}$ NMR spectra. Only one half of each structure is shown for simplicity. 

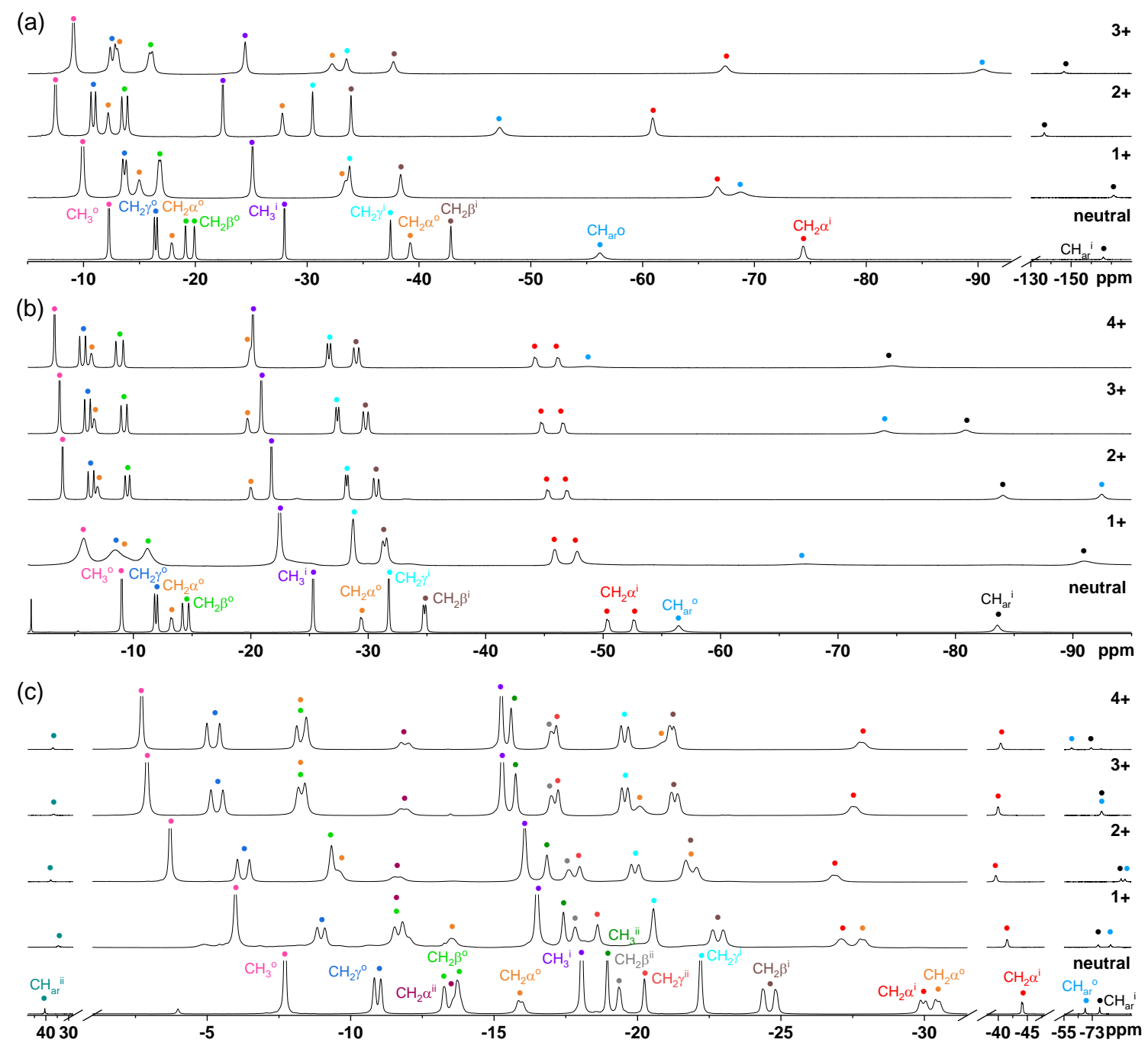

Figure 4. ${ }^{1} \mathrm{H}$ NMR spectra with the assignment of the signals for oxidized and neutral species of (a) [3], (b) [4] and (c) [5] at $295 \mathrm{~K}$. Assignment is described in the text above, more details are given in the Supporting Information. 
(a)

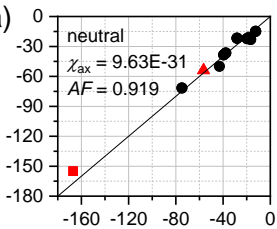

(b) 0 neutral

$-20-\begin{aligned} & \text { neutral } \\ & \chi_{\mathrm{ax}}=1.17 \mathrm{E}-30\end{aligned}$

을 $-40-A F=0.897$

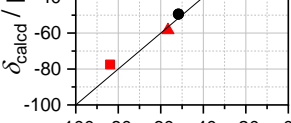

$\begin{array}{llllll}-100 & -100 & -80 & -60 & -40 & -20\end{array}$

(c)

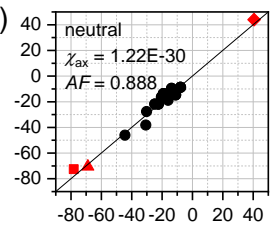

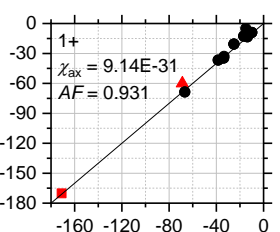
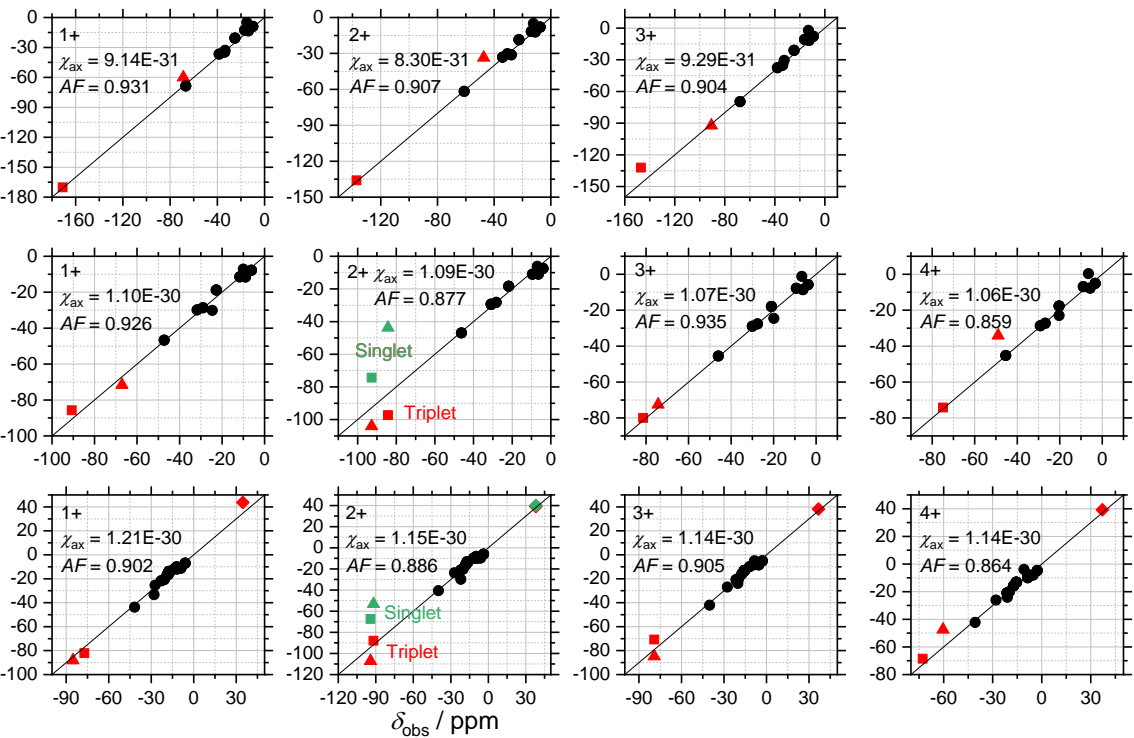

Figure 5. Determination of $\chi_{\text {ax }}$ values for (a) [3], (b) [4] and (c) [5] using observed ( $\left.\delta_{\text {obs }}\right)$ and calculated $\left(\delta_{\text {calcd }}{ }^{1} \mathrm{H}\right.$ NMR signals. Black circle points $(\bullet)$, showing the signals of butoxy protons, were used for least square fit for minimizing the $\Sigma\left(\delta_{\text {calcd }}-\delta_{\text {obs }}\right)^{2}$. The agreement factors $(A F)$ were calculated as $A F$ $=1-\left[\Sigma\left(\delta_{\text {calcd }}-\delta_{\text {obs }}\right)^{2} / \delta_{\text {obs }^{2}}\right]^{1 / 2}$, using all ${ }^{1} \mathrm{H}$ signals, including $\mathrm{CH}_{\mathrm{ar}}{ }^{\mathrm{i}}(\boldsymbol{\square})$ and $\mathrm{CH}_{\mathrm{ar}}{ }^{\mathrm{o}}(\boldsymbol{\Delta})$. Solid black lines on the graphs are the guides showing $\delta_{\text {calcd }}=\delta_{\text {obs }}$ region. The green points on graphs for $[4]^{2+}$ and $[5]^{2+}$ indicate the chemical shifts of the aromatic ${ }^{1} \mathrm{H}$ for the singlet states (without considering the $\delta_{\mathrm{LFC}}$ contributions from triplet states).
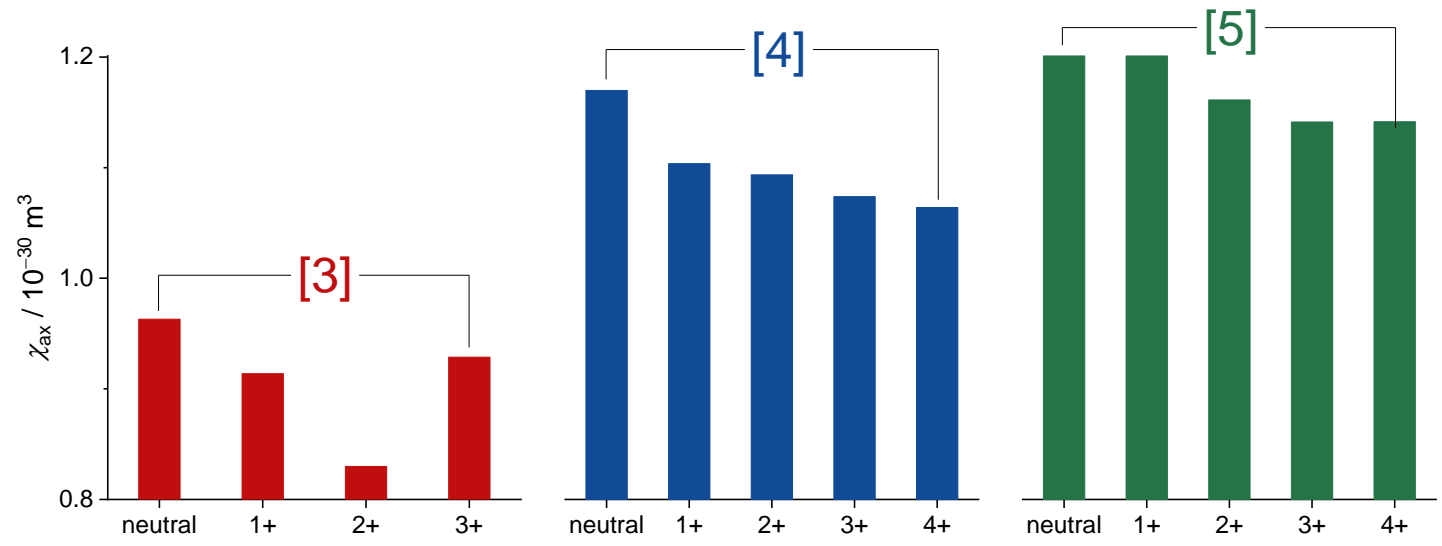

Figure 6. $\chi_{\mathrm{ax}}$ values of (a) [3], (b) [4] and (c) [5] series derived by the ${ }^{1} \mathrm{H}$ NMR analysis at $295 \mathrm{~K}$.

Another point to be mentioned is the paramagnetic shift of $\mathrm{CH}_{\mathrm{ar}}{ }^{\mathrm{o}}$ protons in $[4]^{2+}$ and $[5]^{2+}$ (Figure 5). Since those two complexes have an even number of charges, the ligand $\pi$-radical and resulting $\delta_{\mathrm{LFC}}$ are expected to be absent, only $\delta_{\mathrm{MPC}}$ being the source of the paramagnetic shift. However, the observed chemical shifts for $\mathrm{CH}_{\mathrm{ar}}{ }^{\mathrm{o}}$ in $[4]^{2+}(-92.74 \mathrm{ppm})$ and $[5]^{2+}(-94.2 \mathrm{ppm})$ are about twice as large as the expected $\delta_{\mathrm{MPC}}\left(-44.32 \mathrm{ppm}\right.$ for $[4]^{2+}$ and $-55.65 \mathrm{ppm}$ for $\left.[5]^{2+}\right)$. These discrepancies are solved by 
adding the $\delta_{\mathrm{LFC}}$ derived by the DFT calculations for triplet states of $[4]^{2+}$ and $[5]^{2+}$ to the $\delta_{\mathrm{obs}}^{a r}$, affording the $-102.9 \mathrm{ppm}$ and $-105.9 \mathrm{ppm}$ for $[4]^{2+}$ and $[5]^{2+}$, respectively, which are close to the experimental chemical shifts. The remaining differences of the order of $-10 \mathrm{ppm}$ are mostly due to the contributions of the organic radicals, as shown previously by a more elaborate analysis. ${ }^{79-82}$

\section{Biradical properties of $[4]^{2+}$ and $[5]^{2+}$}

According to the NMR analysis, $[4]^{2+}$ and $[5]^{2+}$ appear to have the $\pi$-radicals on the ligands, especially on the obPc ${ }^{\circ}$. However, the relatively sharp ${ }^{1} \mathrm{H}$ NMR signals in $[4]^{2+}$ and $[5]^{2+}$ (enabling us to acquire ${ }^{1} \mathrm{H}-{ }^{1} \mathrm{H}$ COSY spectra) compared to those in other radical complexes imply the contributions of singlet ( $\pi$-radical silent) states as well. To evaluate the existence of $\pi$-radicals, we performed the electron spin resonance (ESR) for Y(III) analogous of oxidized complexes, which were prepared by the bulk electrolysis in $\mathrm{CH}_{2} \mathrm{Cl}_{2}$ and their formations were confirmed by checking that the absorption spectra of $\mathrm{Y}$ (III) analogous are identical to those of $\mathrm{Tb}(\mathrm{III})$ complexes (Figure S334). Even though Y(III) analogues of $[4]^{2+}$ and $[5]^{2+}$ have even numbers of charges, these complexes are ESR active at room temperature and the $g$-values of them are close to 2, which is common in organic radicals. By cooling down to $80 \mathrm{~K}$, both $[4]^{2+}$ and $[5]^{2+}$ show complicated ESR signals being characteristic for a triplet biradical with an axial zero-field splitting parameter $D$, in contrast to other monoradical species (Figure S330, S331 and S332). The sign of the $D$ values for $[4]^{2+}$ and $[5]^{2+}$ were not determined from the simulation. $|D|$ values of $[4]^{2+}\left(0.0032 \mathrm{~cm}^{-1}\right)$ is slightly larger than that of $[5]^{2+}\left(0.0029 \mathrm{~cm}^{-1}\right)$, indicating that the $[4]^{2+}$ has stronger dipole-dipole interactions between unpaired electrons than $[5]^{2+}$ does because of the smaller $\pi$-extended system in former compound. Focusing on the shape and energy of molecular orbitals in the even-charged complexes, HOMO-LUMO gaps $\left(\Delta E_{\text {LUмо-номо }}\right)$ in $[4]^{2+}$ and $[5]^{2+}(0.20$ and $0.14 \mathrm{eV}$, respectively) are considerably smaller than those in others ( 0.47 to 1.21 $\mathrm{eV}$ ) because HOMO and LUMO in $[4]^{2+}$ and $[5]^{2+}$ are non-bonding orbitals whose shapes are similar

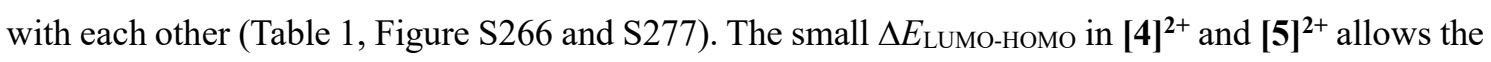
electrons to occupy the LUMO with small energy which results in the formation of biradical states. To get deeper insight into biradical states, we performed natural orbital (NO) analysis for antiferromagnetic (AFM) states using (U)B3LYP/6-31G*83-87 with Stuttgart RSC 1997 ECP $^{88-89}$ for Y and $\mathrm{Cd}$. The occupancies for the lowest unoccupied natural orbitals (LUNO) for singlet states $[4]^{2+}$ and $[5]^{2+}$ were 0.922 and 0.961 , respectively. In addition, diradical character $y$ values for $[4]^{2+}$ and $[5]^{2+}$ are 0.845 and 0.922 , respectively, indicating the contribution of triplet ferromagnetic (FM) states. The calculated exchange interactions between the two $S=1 / 2$ spins among $[4]^{2+}$ and $[5]^{2+}$ are -34 $\mathrm{cm}^{-1}$ and $-3 \mathrm{~cm}^{-1}$, respectively, their magnitudes being small enough so that the triplet (ferromagnetic) states are thermally accessible at liquid nitrogen temperature. These results are consistent with the triplet biradical states observed using ESR measurements at $80 \mathrm{~K}$. In addition, magnitude of $J$ values associates with the length of $\pi$-columns and the magnitude of $|D|$ values. 
Table 1. HOMO and LUMO energies for multiple-decker complexes with even numbers of charges. The energies are derived by the single-point calculations for optimized singlet structures using TPSSh/Def2-SVP.

\begin{tabular}{lcccccccc}
\hline & {$[\mathbf{3}]^{0}$} & {$[\mathbf{3}]^{2+}$} & {$[\mathbf{4}]^{\mathbf{0}}$} & {$[\mathbf{4}]^{2+}$} & {$[\mathbf{4}]^{4+}$} & {$[\mathbf{5}]^{\mathbf{0}}$} & {$[\mathbf{5}]^{2+}$} & {$[\mathbf{5}]^{4+}$} \\
\hline HOMO level / eV & -4.12 & -8.00 & -4.10 & -7.38 & -11.28 & -4.06 & -6.99 & -10.37 \\
LUMO level / eV & -2.91 & -7.48 & -2.90 & -7.18 & -7.18 & -2.93 & -6.85 & -9.90 \\
$\Delta E_{\text {LUмо-номо / eV }}$ & 1.21 & 0.52 & 1.20 & 0.20 & 4.10 & 1.13 & 0.14 & 0.47 \\
\hline
\end{tabular}

Table 2. Comparison of exchange parameters for biradical species of phthalocyaninato and porphyrinato analogues.

\begin{tabular}{cc}
\hline & $J / \mathrm{cm}^{-1}$ \\
\hline$[\mathbf{4}]^{2+}$ & $-34(\mathrm{DFT})$ \\
{$[\mathbf{5}]^{2+}$} & $-3(\mathrm{DFT})$ \\
{$\left[\mathrm{K}_{4}\left\{\mathrm{Lu}\left(\mathrm{CR}{ }_{4} \mathrm{Pc}\right) \mathrm{Pc}\right\}_{2}\right]^{4+}$} & $-0.35(\text { empirical })^{90}$ \\
Pc-Y-FPc-Y-Pc & $-207.4(\mathrm{DFT}),{ }^{79}-93$ (experimental $^{91}$ \\
Por-Y-FPor-Y-Por & $-487(\text { experimental })^{92}$ \\
\hline
\end{tabular}

So far biradical compounds with Pc and Por have been synthesized by connecting two neutral doubledecker monoradical complexes and their $J$ values are summarized in Table 2. ${ }^{79,90-94}$ Among them, triplet state has been reported on $\mathrm{K}^{+}$ion induced supramolecular tetramer $\left[\mathrm{K}_{4}\{\mathrm{Lu}(\mathrm{CR} 4 \mathrm{Pc}) \mathrm{Pc}\}_{2}\right]^{4+}$ composed of crown-substituted double-decker complexes reported by Ishikawa et al, where the four phthalocyaninato ligands align along the $\mathrm{C}_{4}$ axis to make the purely longitudinally extended $\pi$-system. In contrast, connecting the two double-decker complexes by in-plane $\pi$-extended ligand such as fusedphthalocyanine FPc or fused-porphyrin FPor results in the formation of singlet biradical species showing strong antiferromagnetic interactions between $S=1 / 2$ spins. Weak $J$ for $[4]^{2+}$ and $[5]^{2+}$ in the present study are similar to that of $\left[\mathrm{K}_{4}\left\{\mathrm{Lu}\left(\mathrm{CR}_{4} \mathrm{Pc}\right) \mathrm{Pc}\right\}_{2}\right]^{4+}$. It is obvious that the $J$ tends to be weak when the biradicals are composed only by $\pi-\pi$ stacks. These results clearly reflect the differences in orthogonality of interligand orbital overlap, which is retained in the longitudinally extended $\pi$-system with wide interligand stacking angles $\theta$. By combining the in-plane and longitudinal extensions, we can modulate the magnitude of $J$.

\section{X-ray structural analysis}

Among the series of multiple-decker complexes, we have succeeded in getting the crystal structures of anionic $[2]^{-}$containing TBA and series of oxidized complexes, i.e. $[2]^{+},[3]^{2+},[4]^{2+},[4]^{4+},[5]^{4+}$, as summarized in Figure 7 and Figure 8. The charges of the multiple-decker complexes were estimated by counting the number of counter anions $\left(\mathrm{SbCl}_{6}^{-}\right)$per multiple-decker unit. In addition, UV-Vis 
spectra of the crystals dissolved in $\mathrm{CH}_{2} \mathrm{Cl}_{2}$ are identical to those shown in Figure 2, confirming that the charges of the molecules obtained from crystal structure analysis are consistent with electrochemically synthesized complexes. It is obvious that all multiple-decker complexes show bowlshaped distortions on the outer obPc ligands induced through oxidations, in agreement with predictions from DFT calculations.

\section{Crystal structures in [2] series}

The crystal structures of the anionic, neutral and cationic forms of porphyrinato double-decker complexes have been reported recently by Yamashita et $a l .{ }^{49}$ They have reported that the porpnyrinatoporphyrinato distances decrease though oxidations. Similar behavior was observed in multiple-decker series studied here. The obPc-obPc distances $\left(R_{\mathrm{obPc}-\mathrm{obPc}}\right)$ of $[2]^{-}$and $[2]^{+}$are 2.853 and $2.767 \AA$, respectively, supporting the observation that the longitudinal compression occurs by oxidation as reported by Yamashita et al. ${ }^{49}$ The $R_{\mathrm{obPc}-\mathrm{obPc}}$ of $[2]^{0}$ is an intermediate between that of [2] $]^{-}$and $[2]^{+}$. The detailed mechanism of these structural changes is utilized for enhancing SMM properties ${ }^{95-97}$ and controlling the energy barrier for ligand rotation. ${ }^{98}$. HOMO of $[2]^{-}$is an antibonding $\pi$-orbital which has a node between the obPc ligands. The oxidation reactions remove the electrons from the antibonding HOMO of $[2]^{-}$, increasing the bond order between the obPc ligands and decreasing the distance $R_{\mathrm{obPc}-\mathrm{obPc}}$. The short $R_{\mathrm{obPc}-\mathrm{obPc}}$ of [2] series compared to those of other multiple-decker complexes enhances the steric hindrance between obPc ligands, causing the wide stacking angles of obPcs holding metal ions $\left(\theta_{\mathrm{obPc}} \sim 45^{\circ}\right)$ at which the interligand steric hindrance is minimized. The crystal packing of [2] series is affected by both the counter ions and the solvent molecules. [2] ${ }^{-}$ contains $\mathrm{TBA}^{+}$and DMSO as the counter cation and crystal solvent, respectively. The flexible butyl groups of $\mathrm{TBA}^{+}$enable these to adopt a flat structure so that they are stacked alternatively with $[2]^{-}$ units, thus constructing the slipped column packing along the $c$-axis. In $[2]^{+}$, slipped column packing consists of $[2]^{+}$units and toluene molecules is surrounded by $\mathrm{SbCl}_{6}{ }^{-}$because of the spherical shape of $\mathrm{SbCl}_{6}{ }^{-}$making it hard to stack alternatively with $[2]^{+}$units.

\section{Crystal structures in [3] series}

Crystal structures of $[3]^{0}$ and $[3]^{2+}$ are shown in Figure 7(b). In both complexes, the two $\mathrm{Tb}(\mathrm{III})$ are crystallographically equivalent and are connected by an inversion operation. In $[3]^{0}, \theta_{\mathrm{obPc}}, R_{\mathrm{obPc}-\mathrm{obPc}}$ and intramolecular Tb-Tb distance $\left(R_{\mathrm{Tb}-\mathrm{Tb}}\right)$ are $31.77^{\circ}, 3.047 \AA$ and $3.517 \AA$, respectively. Oxidation to the $[3]^{2+}$, which corresponds to the removal of two electrons from an antibonding HOMO of $[3]^{0}$, makes the $R_{\mathrm{obPc}-\mathrm{obPc}}$ and the $R_{\mathrm{Tb}-\mathrm{Tb}}$ shorter $(2.980 \AA$ and $3.435 \AA$, respectively), resulting in the wide $\theta_{\mathrm{obPc}}\left(44.21^{\circ}\right)$ in order to avoid the steric interaction between the phenyl rings of obPcs. Due to the large interligand steric effect induced by longitudinal compression, the outer obPcs of $[3]^{2+}$ show bowlshaped distortions. Similar structural changes are predicted by the geometry optimization of $[3]^{2+}$ at the TPSSh/Def2-SVP level (Figure S253). ${ }^{62-64,}{ }^{99}[3]^{2+}$ constructs the slipped column structure surrounded by $\mathrm{SbCl}_{6}{ }^{-}$ions extended along the $a$-axis, as seen in $[2]^{+}$. 


\section{Crystal structures of [4] series}

In case of [4] series, we have succeeded in getting the crystal structures of $[4]^{2+}$ and $[4]^{4+}$, whereas the structure $[4]^{0}$ was previously reported. The space groups of $[4]^{2+}$ and $[4]^{4+}$ are $P 4 / \mathrm{ncc}$ and $P 4 / \mathrm{nmm}$, respectively, and four-fold axes exist along $\mathrm{Tb}-\mathrm{Cd}$ - $\mathrm{Tb}$ lines ( $c$-axes). Due to the positional disorder of two obPcs of $[4]^{4+}$, giving the identical geometries in each disordered state, one representative geometry is shown in Figure 7(c). Same as with the other multiple-decker series, [4] series show bowlshaped distortions on the outer obPc ligands induced by oxidations. In [4] series, although two $\mathrm{Tb}$ (III) ions are crystallographically inequivalent, the $\theta_{\mathrm{obPc}}$ values of two Tb1 and Tb2 (Figure 7(c)) of [4] ${ }^{0}$ are similar with each other $\left(22.32^{\circ}\right.$ and $\left.23.65^{\circ}\right)$. In $[4]^{2+}, \theta_{\mathrm{obPc}}$ of $\mathrm{Tb} 1$ and $\mathrm{Tb} 2$ are $42.27^{\circ}$ and $38.68^{\circ}$, respectively. Unexpectedly, differences in $\theta_{\mathrm{obPc}}$ are further enhanced in $[4]^{4+}$, in which two kinds of $\theta_{\mathrm{obPc}}\left(45.00^{\circ}\right.$ for $\mathrm{Tb} 1$ and $16.54^{\circ}$ for $\left.\mathrm{Tb} 2\right)$ are seen. In other words, square antiprismatic (SAP) and square prismatic (SP) geometries exist in one $[4]^{4+}$ unit. Considering that the $[4]^{4+}$ dissolved in $\mathrm{CD}_{2} \mathrm{Cl}_{2}$ shows a limited number of ${ }^{1} \mathrm{H}$ signals due to the symmetric structure of $[4]^{4+}$, the asymmetric structure of $[4]^{4+}$ seen in SXRD seems to be induced by the steric effects from the solvent molecules and $\mathrm{SbCl}_{6}{ }^{-}$ incorporated into the crystal. The geometry optimization for $[4]^{4+}$, which estimates the structure of $[4]^{4+}$ in gas phase, affords the symmetric structure, further supporting the importance of intermolecular forces in the crystals for asymmetric conformation seen in SXRD. Although it is a preliminary result, we got the incomplete crystal structure of $[4]^{4+}$ crystalized by toluene and revealed that the SAP and SP geometry coexist in one molecule (Figures S324 and S325), indicating that asymmetric conformation of $[4]^{4+}$ in the crystal a universal property. In the structure of $[4]^{4+}$ with toluene, SP conformations are stabilized by the toluene molecules incorporated into the grooves of obPcs. Although the position of solvent molecules of $[4]^{4+}$ with TBT/TCE could not be determined due to heavy disorder, the residual electron densities in the grooves around SP structure imply that solvent incorporation stabilizes SP geometry.

In contrast to previous examples ([2], [3] series and $\left.[4]^{0}\right),[4]^{2+}$ and $[4]^{4+}$ show $1 \mathrm{D}$ column packing along $c$-axes because the long molecular structures of them are disfavored for the formation of slipped columns. In addition, oxidations of $[4]^{0}$ induce the bowl-shaped distortions of outer obPcs, including $n$-butoxy chains, impeding the construction of intermolecular $\pi-\pi$ stack structures. In addition, the 1D packing is seen in preliminary SXRD results of $[4]^{4+}$ prepared in toluene. Therefore, we speculate that the long molecular structure and the absence of intermolecular $\pi-\pi$ stacks cause the construction of 1D structures, as seen in $[4]^{2+}$ and $[4]^{4+}$.

In $[4]^{2+}$, column packing which consists of $[4]^{2+}$ units and tert-butyl toluene (TBT) molecules is surrounded by $\mathrm{SbCl}_{6}{ }^{-}$anions. The shortest intermolecular $\mathrm{Tb}-\mathrm{Tb}$ distance and pitch of the column are $9.937 \AA$ and $16.480 \AA$, respectively. The intermolecular face-to-face dimension of outer obPcs is 7.294 $\AA$, which is longer compared to those of $[2]^{+},[3]^{2+}$ and $[4]^{0}$ because the bowl-shaped distortion on outer obPcs and solvent molecules (TBT) prevent formation of the intermolecular $\pi-\pi$ stacks. 
Assembly of 1D columns along the $c$-axis provides a square lattice as well as 1D voids extending along the $c$-axis. The observation of electron densities in these voids indicates that they not empty but filled with disordered solvent molecules.

In $[4]^{4+}, 1 \mathrm{D}$ column packing along $c$-axis, which consists of $[4]^{4+}$ units and $\mathrm{SbCl}_{6}{ }^{-}$anions, was observed. The two additional $\mathrm{SbCl}_{6}{ }^{-}$compared to $[4]^{2+}$ are incorporated into the voids made by strongly bowl-shape distorted outer ligands of $[4]^{4+}$. As a result, the intermolecular Tb-Tb distance and the pitch of the column increase to $12.917 \AA$ and $19.410 \AA$, respectively. Obviously, these two $\mathrm{SbCl}_{6}{ }^{-}$ act as the spacer to increase the intermolecular distances. The remaining two $\mathrm{SbCl}_{6}{ }^{-}$anions in $[4]^{4+}$ surround the $1 \mathrm{D}$ columns such as the $\mathrm{SbCl}_{6}{ }^{-}$of $[4]^{2+}$. As similar to $[4]^{2+}, 1 \mathrm{D}$ column packing of $[4]^{4+}$ assembles to square lattice, making the $1 \mathrm{D}$ voids along $c$-axis which should be filled with disordered solvent molecules. Furthermore, the similar 1D packing and voids are seen in the incomplete structure of $[4]^{4+}$ with toluene molecules, indicating that the packing of $[4]^{4+}$ is controlled by the molecular structure of [4] and the number of counter ions rather than the solvent molecules.

\section{Crystal structures of [5] series}

In case of [5] series, we have succeeded in getting the structure of [5] ${ }^{4+}$. In contrast to the wave-like distortion of obPcs in $[5]^{0}$, outer obPcs of $[5]^{4+}$ show bowl-shaped distortions due to longitudinal compression effects induced by the oxidation. Although $\mathrm{Tb}(\mathrm{III})$ ions of $[5]^{0}$ are crystallographically equivalent with each other, two kinds of $\mathrm{Tb}(\mathrm{III})$ ions, one of which being of SAP and the other being of SP coordination geometry, were observed in $[5]^{4+}$, as seen for $[4]^{4+}$. It is obvious that the solvent molecules (benzene) located in the grooves formed by the ligands stabilize the SP geometry (Figure 7(d)). Alternative stacking of [5] ${ }^{4+}$ units and one disordered $\mathrm{SbCl}_{6}{ }^{-}$generates $1 \mathrm{D}$ column packing along the $a$-axis as seen in the crystal structures of $[4]^{4+}$. We also succeeded in getting the incomplete structures of $[5]^{4+}$ containing toluene molecules by the slow diffusion using $\mathrm{CH}_{2} \mathrm{Cl}_{2}$ and toluene (Figures S326 and S327), where [5] units containing SP and SAP geometries construct the 1D column packing along $c$-axis. Furthermore, the SP geometry is stabilized by the toluene molecules incorporated to the groove of SP geometries, showing the importance of solvent molecules for the coexistence of SP and SAP geometries in one multiple-decker unit.

\section{Summary for the crystal structures and packing of the oxidized multiple-decker complexes}

In summary, all the multiple-decker complexes show axial compression by the oxidations as indicated by DFT calculations. The HOMOs of the neutral multiple-decker complexes are antibonding orbitals showing nodes between obPcs. Oxidation reactions remove electrons from antibonding HOMOs, increasing the bond orders between obPcs. As a result, longitudinal compression induced by the oxidations was observed by the X-ray analysis. However, the existence of two kinds of geometry around $\mathrm{Tb}(\mathrm{III})$ ions (SAP and SP) in $[4]^{4+}$ and $[5]^{4+}$ were not expected from theoretical calculations. The SP geometries seem to be stabilized by the interactions between the solvent molecules and counter ions. 
The XRD analyses show that the crystal packing of the multiple-decker complexes is dependent on the length of the molecules, distortion on outer obPcs, the numbers and arrangements of the counter ions and co-crystallized solvent molecules. Short molecular heights of [2] and [3], as well as the planarity of outer obPcs seen in neutral multiple-decker complexes permit the slipped column packing. In contrast, 1D linear column packing is favored in the longer molecules such as [4] and [5] series. In addition, $\mathrm{TBA}^{+}$anions and $[2]^{-}$stack alternately and construct columns because of the flat structure of $\mathrm{TBA}^{+}$. The octahedral structure of $\mathrm{SbCl}_{6}{ }^{-}$makes these surround the column packing as seen in $[2]^{+}$, $[3]^{2+}$ and $[4]^{2+}$, instead of being incorporated inside the column structure. This relationship is violated when the excess of $\mathrm{SbCl}_{6}{ }^{-}$exist as seen in $[4]^{4+}$ and $[5]^{4+}$, where the $\mathrm{SbCl}_{6}{ }^{-}$and multiple-decker units construct 1D column packing by stacking alternatively. The bowl-shaped distortions on outer obPc of highly oxidized species also take a vital role in creating the space to be filled by $\mathrm{SbCl}_{6}{ }^{-}$ions and the thus resulting 1D packing. Keeping in mind the magnetic properties discussed below, and the existing knowledge about the sensitivity of the magnetic properties of similar SMMs to the coordination environment ${ }^{100-102}$, we underline the conclusion that not only the molecular structure, but also the packing of molecules in the crystal play an almost equally important role in lanthanidephthalocyaninato based SMMs. We predict that there is much to be achieved from systematically varying and strictly controlling crystal growth mechanisms of future SMMs, especially when under exposure of magnetic and electric fields. ${ }^{103-108}$ 
(a)

(c)
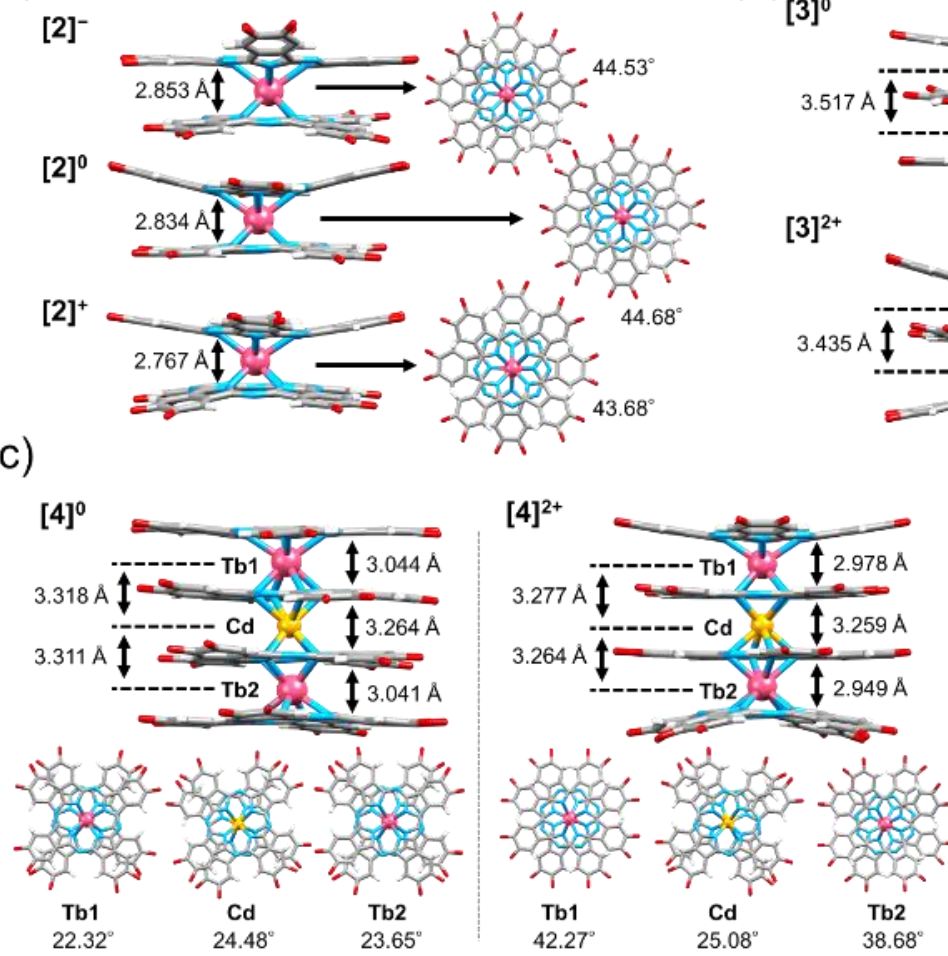

(b)

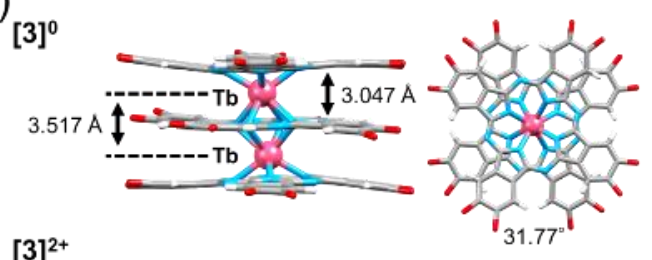

$[3]^{2+}$
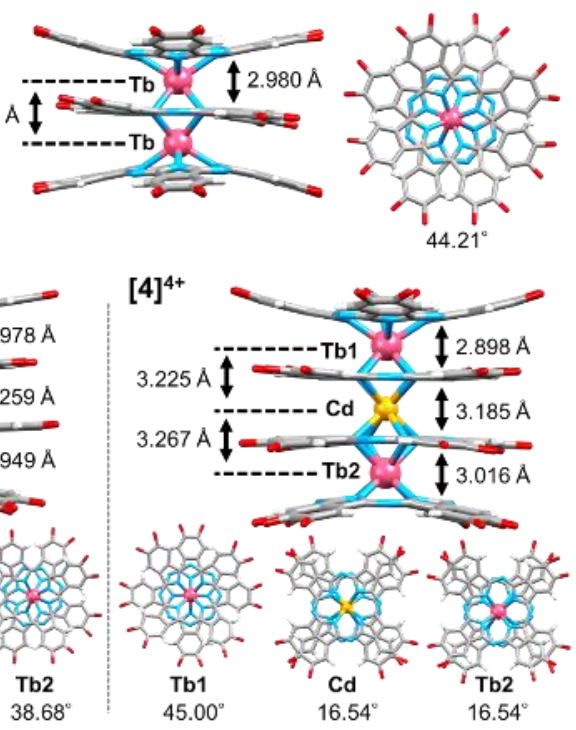

(d)
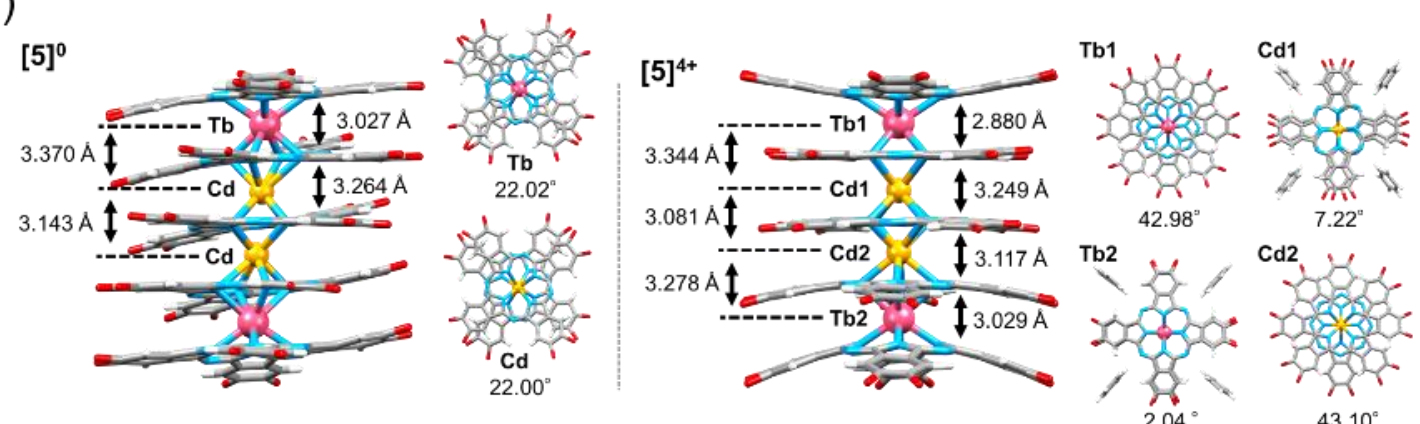

Figure 7. Comparison of molecular structures of (a) [2], (b) [3], (c) [4] and (d) [5] series. Numbers in the figures indicate the $R_{\mathrm{obPc}-\mathrm{obPc}}$ and $\theta_{\mathrm{obPc}} . r_{\mathrm{obPc}}$ are the distances between centroids coordinating four coordinating $\mathrm{N}$ atoms of obPcs. $\theta_{\mathrm{obPc}}$ are averaged torsional angles. Butyl chains and counter anions $\left(\mathrm{SbCl}_{6}{ }^{-}\right)$were omitted for clarity. Color coding: pink $=\mathrm{Tb}$, yellow $=\mathrm{Cd}$, red $=\mathrm{O}$, blue $=\mathrm{N}$, gray $=\mathrm{C}$, white $=\mathrm{H}$. 
(a)

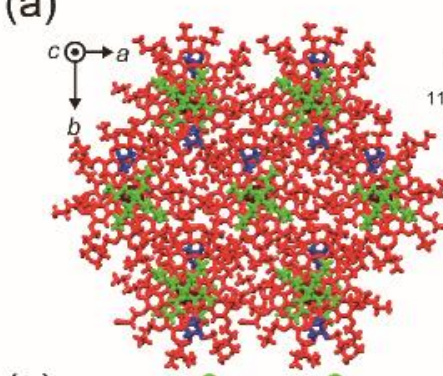

(c)

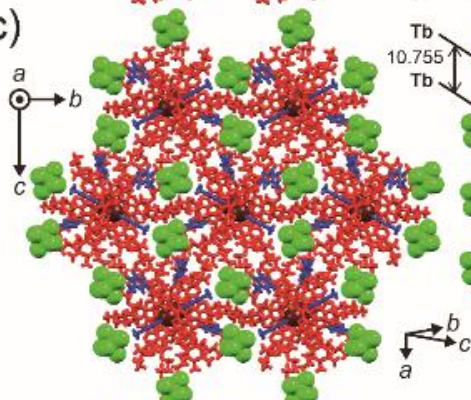

(e)

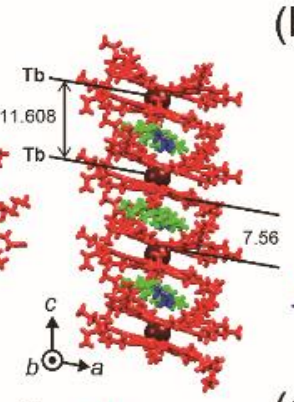

(b)
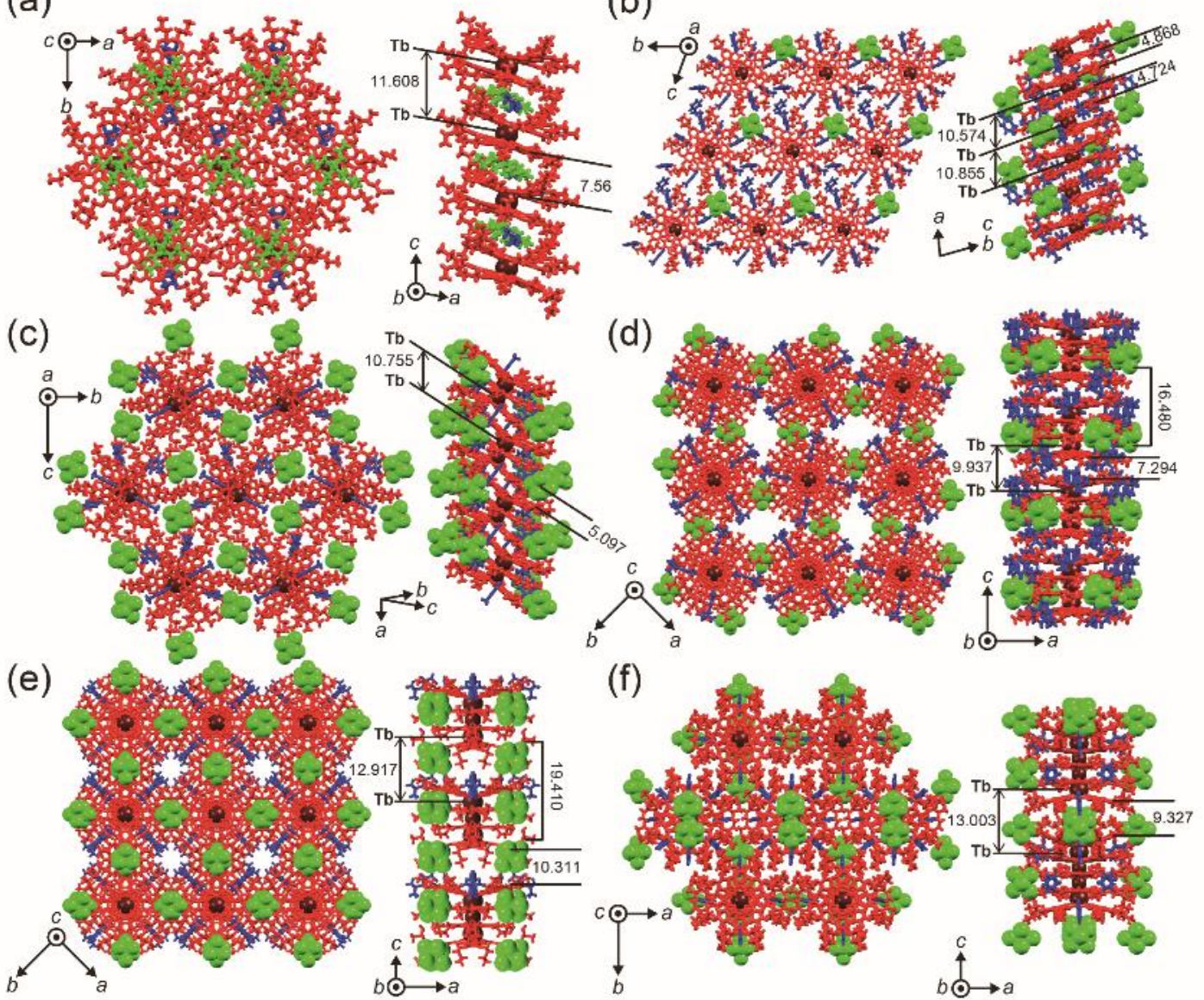

Figure 8. Crystal packing of (a) $[2]^{-}$, (b) $[2]^{+}$, (c) $[3]^{2+}$, (d) $[4]^{2+}$, (e) $[4]^{4+}$ and (f) $[5]^{4+}$. Multiple-decker complexes, metal centers ( $\mathrm{Tb}$ or $\mathrm{Cd}$ ), counter ions $\left(\mathrm{TBA}^{+}\right.$for $[2]^{-}$and $\mathrm{SbCl}_{6}{ }^{-}$for others) and solvent molecules are shown in red, brown, green and blue respectively. List of solvent molecules: DMSO; (a), toluene; (b) and (c), TBT; (d) and (e), benzene; (f). The numbers in the figure show intermolecular $\mathrm{Tb}$ - $\mathrm{Tb}$ distances and inter ligand distances (distances between $\pi$-planes) respectively in units of $\AA$. In (d) and (e), the pitch of the columns is indicated in $\AA$.

\section{Magnetic properties}

The magnetic properties of oxidized phthalocyaninato double-decker SMMs have been reported by Ishikawa et al., where oxidation of phthalocyaninato double-decker complexes increases the activation energy for spin reversal $(\Delta E)$ due to the decrease in inter-ligand distances. ${ }^{95-97} \mathrm{X}$-ray structural analysis for [2] series in the present research supports the decrease in $R_{\mathrm{obPc}-\mathrm{obPc}}$ with increasing the oxidation numbers.

\section{Magnetic properties for [2]}

The DC magnetic measurements for $[2]^{-}$and $[2]^{+}$are summarized in Figure S1 and S5, respectively. The hump below $15 \mathrm{~K}$ seen in $[2]^{-}$is assumed to relate to magnetization blocking of [2] $]^{-}$. In case of $[2]^{+}$, an increase in $\chi_{\mathrm{M}} T$ values was observed below $10 \mathrm{~K}$ due to intermolecular ferromagnetic 
interactions. Details of the electronic structures of [2] series were obtained from ab initio calculations using the crystal structure coordinates of [2] series with modifying the $n$-butoxy groups to methoxy ones in input files. The experimental and theoretical $\chi_{\mathrm{M}} T$ vs $T$ plots for [2] series are in agreement with each other. Introduction of a mean-field parameter $z J=0.02 \mathrm{~cm}^{-1}$ reproduces the increase in $\chi_{\mathrm{M}} T$ values in $[2]^{+}$, thus validating the theoretical calculations. All [2] species show strong axial magnetic anisotropy as reported previously $35-37,40-41,102$, where the ground doublet is composed by almost pure $\mid \pm 6>$ states, first excited $\mid \pm 5>$ states being above $300 \mathrm{~cm}^{-1}$ in energy (Figure 9). To investigate the SMM properties of [2] series, we performed ac magnetic measurements for $[2]^{-}$and $[2]^{+}$with and without a $2 \mathrm{kOe} d \mathrm{c}$ bias field. Both species show clear peaks in $\chi_{\mathrm{M}}$ " (imaginary part of ac magnetic susceptibilities) vs. $v$ (ac frequency) plots without bias a dc magnetic field, as summarized in Figure 10(a). In the presence of the bias field ( $2 \mathrm{kOe})$, the $\chi_{\mathrm{M}}$ " peaks of both species show a small shift to lower $v$ regions, indicating that a bias field quenches quantum tunneling of the magnetization (QTM), due to the Zeeman splitting in the ground doublet. ${ }^{109}$ Evidently, the peak tops of $\chi_{\mathrm{M}}$ " shift to the lower $v$ regions by the oxidation from $[2]^{-}$to $[2]^{+}$, as reported by Ishikawa et al. ${ }^{96}$ All ac magnetic susceptibilities for [2] series were fitted using the generalized Debye model to acquire the spin relaxation time $\tau$ (Figures $\mathrm{S} 2, \mathrm{~S} 3, \mathrm{~S} 6$ and $\mathrm{S} 7$ ). Activation energy for spin reversal $\Delta E$ and frequency factor $\tau_{0}$ with the bias field determined by an Arrhenius fit are $\Delta E=488(5) \mathrm{cm}^{-1}$ and $\tau_{0}=3.4(7) \times$ $10^{-12} \mathrm{~s}$ for $[2]^{-}$and $\Delta E=580(2) \mathrm{cm}^{-1}$ and $\tau_{0}=3.0(2) \times 10^{-13} \mathrm{~s}$ for $[2]^{+}$(Figures S4 and S8). The experimental $\Delta E$ seems to be close to the theoretical energy levels of second excited states $\left(536 \mathrm{~cm}^{-1}\right.$ for $[2]^{-}$and $524 \mathrm{~cm}^{-1}$ for $\left.[2]^{+}\right)$rather than first excited states $\left(325 \mathrm{~cm}^{-1}\right.$ for $[2]^{-}$and $322 \mathrm{~cm}^{-1}$ for $\left.[2]^{+}\right)$. Therefore, in the present [2] series the magnetic relaxations via $\mid \pm 4>$ might occur. It is known that the coincidence of the magnetic easy axes of ground and excited doublets decrease the magnetic transition probabilities and enhance the magnetic relaxation via higher sublevels. According to the ab initio calculations, second exited states of [2] series are composed of almost pure $| \pm 5\rangle$, hence the linearity of magnetic easy axis between the ground, first and second exited states is sustained. Although abinitio calculations indicate that $[2]^{-}$and $[2]^{+}$are similar with each other, experimental $\Delta E$ values of $[2]^{+}$is substantially larger than that of [2] $]^{-}$. In [2] series, a small portion of $\mid \pm 3>$ and $\mid \pm 2>$ mixes to the first excited and second excited doublet $(\mid \pm 5>$ and $\mid \pm 4>)$, respectively. The amount of such mixing in $[2]^{+}$is smaller than that in $[2]^{-}$, enhancing the relaxation via second excited state in $[2]^{+}$.

\section{Magnetic properties for [3]}

In [3], $\chi_{\mathrm{M}} T$ vs $T$ plots show an increase at low temperature due to intramolecular ferromagnetic interactions between $\mathrm{Tb}(\mathrm{III})$ ions. Given the relative positions of two $\mathrm{Tb}(\mathrm{III})$ ions derived by crystal structures and the $a b$ initio derived ligand-field splitting, the contribution of the intramolecular magnetic dipole-dipole interactions between $\mathrm{Tb}(\mathrm{III})$ ions was evaluated. As shown in the Supporting Information, the $\chi_{\mathrm{M}} T$ values in [3] series were fitted by taking into account the magnetic dipole-dipole interactions. As seen in Figure 9, significant easy axis magnetic anisotropy is expected across [3] series 
due to almost pure $\mid \pm 6>$ ground states. Magnetic easy axes of $\mathrm{Tb}$ (III) ions in [3] series are perpendicular to obPc planes, stabilizing a head-to-tail ferromagnetic arrangement of magnetic dipoles. AC magnetic susceptibilities for [3] series at zero dc field show a single peak, which is fitted by using the generalized Debye model. In contrast to [2] series, the peak top of $\chi_{\mathrm{M}}$ " shifts to higher $v$ regions by the oxidations. In other words, the $\tau$ becomes faster with the ligand oxidations. The application of bias dc field scarcely affects the $\chi_{\mathrm{M}}$ " vs $v$ plots of [3], indicating that QTM is suppressed by the intramolecular MD interactions acting as the bias field. ${ }^{61,110-113}$ From the Arrhenius plots at $2 \mathrm{kOe}$, the $\Delta E$ values were confirmed to be $233 \mathrm{~cm}^{-1}$ for [3] $]^{0}$ and $172 \mathrm{~cm}^{-1}$ for [3] $]^{2+}$. Again, $\Delta E$ of [3] becomes smaller by the oxidations, in contrast to observations for the [2] series.
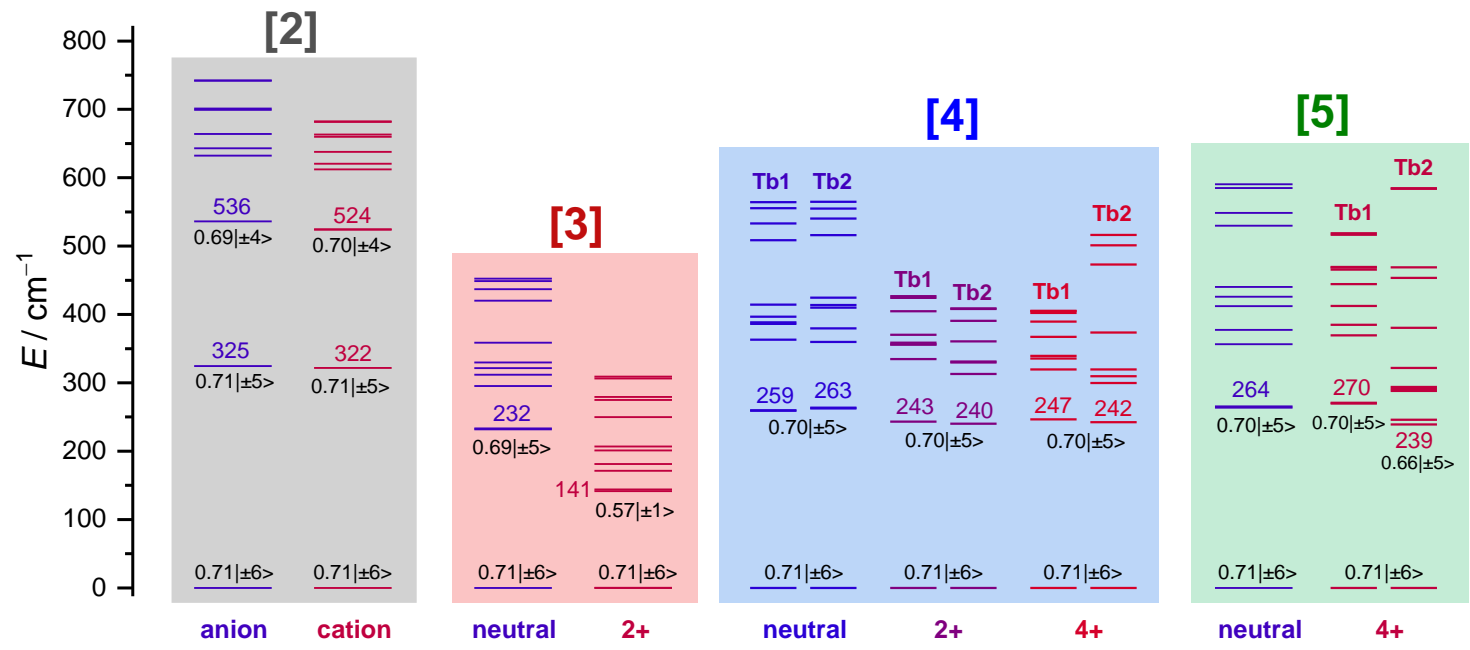

Figure 9. Ligand field splitting for multiple-decker complexes calculated by CASSCF ${ }^{114-118}$ calculations, using the coordination fragment around $\mathrm{Tb}(\mathrm{III})$ ions with 421 of electron pairs for [2] ${ }^{-}$, 420 electron pairs for $[2]^{+}, 455$ electron pairs for $[3]^{0}, 454$ electron pairs for $[3]^{2+}$ and 444 electron pairs for others. Since the $[4]^{0},[4]^{2+}$ and $[4]^{4+}$ and $[5]^{4+}$ contain crystallographically inequivalent $\mathrm{Tb}(\mathrm{III})$ ions, two kinds of diagrams are displayed. Energy differences between ground and first exited doublets, which correspond to $\Delta E$, are shown in $\mathrm{cm}^{-1}$ units. More details are given in the Supporting Information. 
(a)

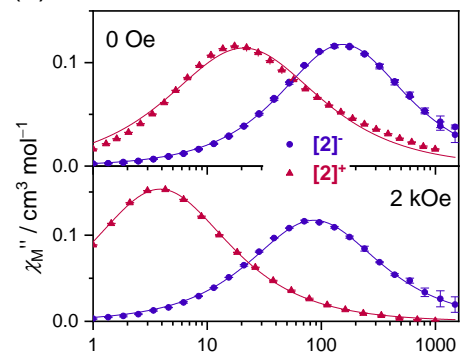

(c)

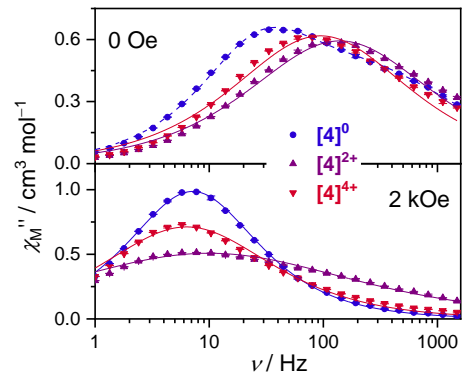

(b)

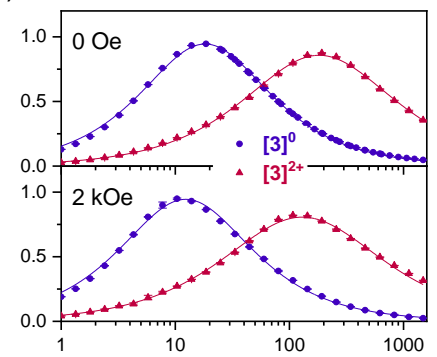

(d)

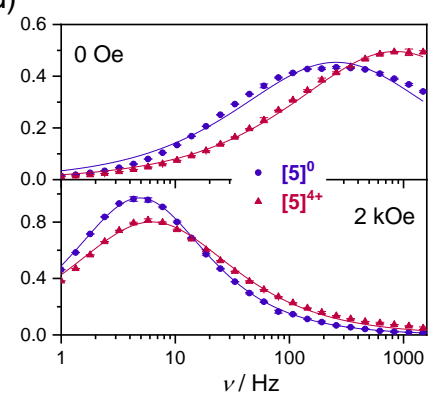

Figure 10. Selected $\chi_{\mathrm{M}}$ " vs $v$ plots for (a) [2] at $35 \mathrm{~K}$, (b) [3] at $10 \mathrm{~K}$, (c) [4] at $10 \mathrm{~K}$ and (d) [5] at 10 $\mathrm{K}$ at $0 \mathrm{Oe}$ (upper) and at $2 \mathrm{kOe}$ (lower) bias field. Solid curves and dotted curves represent fits using the generalized and extended Debye models, respectively.

The experimental $\Delta E$ values of [3] series are similar to the first exited energy levels by the ab initio calculations. In addition, CASSCF calculations support the decrease in $\Delta E$ values by the oxidations from $[3]^{0}$ to $[3]^{2+}$. Significant decrease in $B_{2}^{0}$ term from $322 \mathrm{~cm}^{-1}$ to $149 \mathrm{~cm}^{-1}$ indicates that axial component of ligand field diminishes by the oxidation. Although the $\Delta E$ values should be a function of the large numbers of geometrical parameters, the $\Delta E$ in the present case seems to be dependent on the intramolecular $\mathrm{Tb}-\mathrm{Tb}$ (or Tb-Cd) distances $\left(R_{\mathrm{Tb}-\mathrm{M}} ; \mathrm{M}=\mathrm{Tb}\right.$ or $\mathrm{Cd}$ ) because the approach of the positively charged metal ions to the $\mathrm{Tb}(\mathrm{III})$ center decreases the axial component of negative charges. This trend was investigated by point charge calculations using McPhase $5.2^{119}$ instead of high-cost CASSCF calculations (Supporting Information). The structural models for point charge calculations were prepared by using the coordination geometry of $[3]^{0}$ and the displacement parameters reported by Ariño and Coronado et al. ${ }^{120}$ The charges on coordinating $\mathrm{N}$ atoms and the opposing of $\mathrm{Tb}(\mathrm{III})$ ions are set to $-0.5 \mathrm{e}$ and $+3 \mathrm{e}$, respectively. The energy difference between ground and first excited states is expected to be $242 \mathrm{~cm}^{-1}$, which is in good agreement with the experimental $\triangle E$ and CASSCF results. In [3] series, the oxidation reaction to the $[3]^{2+}$ species induces the longitudinal compression and shortens the $R_{\mathrm{Tb}-\mathrm{Tb}}$. Figure S320 illustrates the linear decrease in the first excited energy with decreasing $R_{\mathrm{Tb}-\mathrm{Tb}}$. When the experimental $R_{\mathrm{Tb}-\mathrm{Tb}}$ of $[3]^{2+}$ is used, the excited energy is $170 \mathrm{~cm}^{-1}$. The good agreement between experimental $\Delta E$ values and point charge results indicate a decrease in $R_{\mathrm{Tb}}$ ть as induced by the oxidations makes a significant impact on the decrease in $\Delta E$. The decrease in the 
magnetic anisotropy as induced by the oxidation is consistent with the decrease in $\chi_{\text {ax }}$ values as determined from NMR analysis.

\section{Magnetic properties for [4]}

In all the [4] series, an increase in $\chi_{\mathrm{M}} T$ values was observed at low $T$ due to ferromagnetic interactions between $\mathrm{Tb}(\mathrm{III})$ ions. The inclusion of intramolecular magnetic dipole-dipole interactions makes it possible to reproduce these increases, indicating the negligible contribution from exchange or intermolecular magnetic interactions. Ab initio ligand field splitting indicates that ground doublets of all the $\mathrm{Tb}(\mathrm{III})$ ions in [4] series are composed of the states $| \pm 6\rangle$, suggesting the strong easy axis magnetic anisotropy on $\mathrm{Tb}(\mathrm{III})$ ions. It is noteworthy that the wavefunctions of the ground $(0.71 \mid \pm 6>)$ and first-excited states $(0.70 \mid \pm 6>)$ are independent on $\theta_{\mathrm{obPc}}$ values, even though it is known that SAP geometries $\left(\theta_{\mathrm{obPc}}=45^{\circ}\right)^{121-122}$ are one of the most promising candidates for enhancing lanthanoid SMM properties. The wavefunctions as well as the first ligand-field splitting energy seem to be affected by coordinating $\mathrm{Cd}(\mathrm{II})$ ions, which cause asymmetric coordination geometries around $\mathrm{Tb}(\mathrm{III})$ ions, rather than $\theta_{\mathrm{obPc}}$ values.

$\chi_{\mathrm{M}}$ " vs $v$ plots for [4] series without bias magnetic fields show unusually broad peaks because of the unquenched quantum tunneling of the magnetization (QTM) in the weak intramolecular magnetic dipole-dipole interactions between $\mathrm{Tb}(\mathrm{III})$. Previously, Katoh et al. have reported that the dual magnetic relaxations are observed in $[4]^{0}$ and $[5]^{0}$ even though the two $\mathrm{Tb}(\mathrm{III})$ ions are equivalent. ${ }^{61}$,

${ }^{123}$ We speculate that ferromagnetic states $\mid \pm 6, \pm 6>$ and antiferromagnetic states $\mid \pm 6, \mp 6>$, which are almost degenerated with each other due to weak coupling, enhance these relaxations. The dual magnetic relaxations and resultant two relaxation times $\tau_{1}$ and $\tau_{2}$ for $[4]^{0}$ were acquired using the extended Debye model which contains two generalized Debye models. As similar to the previous report, slower $T$-dependent $\tau_{1}$ and faster less $T$-dependent $\tau_{2}$ were observed in [4] $]^{0}$. Although similar broadening in $\chi_{\mathrm{M}}$ " vs $T$ plots was seen in $[4]^{2+}$ and $[4]^{4+}$, it was possible to fit the shape of the peak with the generalized Debye model because $\tau_{1}$ and $\tau_{2}$ of these two species are close with each other. As seen in Figure 10, in the absence of a bias field, the peak top $v$ of $[4]^{0}$ is smallest ( $\tau$ is longest) among [4] series. The peak tops of $\chi_{\mathrm{M}}$ " shift to the lower $v$ regions with the application of a $2 \mathrm{kOe}$ dc bias field because of the quenching of QTM induced by Zeeman splitting. At $2 \mathrm{kOe}$, the $[4]^{4+}$ shows longest $\tau$ values. However, the positions of the peak top $v$ are less dependent on oxidation states compared to [2] and [3] series. In addition, the ac magnetic susceptibilities are fitted using the generalized Debye model even though two crystallographically inequivalent $\mathrm{Tb}$ (III) ions exist in all species, being consistent with the ab initio results where the magnetic properties on $\mathrm{Tb}(\mathrm{III})$ ions in [4] series are less affected by variation in the coordination environment compared to [3] series. $\Delta E$ values for at $2 \mathrm{kOe}$ are $237(6) \mathrm{cm}^{-1}$ for $[4]^{0}, 194(9) \mathrm{cm}^{-1}$ for $[4]^{2+}$ and $242(11) \mathrm{cm}^{-1}$ for $[4]^{4+}$. These values are close to the first excited levels in the ab initio derived ligand field splitting.

\section{Magnetic properties for [5]}


The $\chi_{\mathrm{M}} T$ vs $T$ plots of all [5] series show a small increase in the low temperature regions, indicating the ferromagnetic interactions between $\mathrm{Tb}(\mathrm{III})$ ions. Ab initio ligand-field splitting and the inclusion of intramolecular magnetic dipole-dipole interactions reproduce the $\chi_{\mathrm{M}} T$ vs $T$ plots of [5] series, indicating the negligible contribution from the intermolecular magnetic interactions. Both species show clear $v$ dependences in ac magnetic susceptibilities without a bias dc field. However, the application of a bias field makes the $\tau$ much slower due to suppression of QTM. The magnetic dipole interactions in [5] series is too weak to fully suppress the QTM, in contrast to [3] series of which ac susceptibilities are scarcely affected by a bias field. The single but broad peaks are seen in $\chi_{\mathrm{M}}$ " vs $v$ plots for [5] series and these arise from the almost degenerated $\mid \pm 6, \pm 6>$ and $\mid \pm 6, \mp 6>$ as a result of the weak $\mathrm{Tb}(\mathrm{III})-\mathrm{Tb}(\mathrm{III})$ interactions. In the presence of a bias field, these dual magnetic relaxations become a sharp single relaxation that could be fitted by the generalized Debye model, as seen in $[4]^{0}$ and previous results. ${ }^{61}$ The $\Delta E$ and $\tau_{0}$ values of [5] ${ }^{0}$ with the bias fields were $251(6) \mathrm{cm}^{-1}$ and $2.9(7)$ $\times 10^{-9} \mathrm{~s}$, respectively. The experimental $\Delta E$ and first exited energy of ab initio ligand field levels are similar with each other. The ac magnetic susceptibilities for $[5]^{4+}$ are similar to those for $[5]^{0}$. An Arrhenius fit at $2 \mathrm{kOe}$ affords the $\Delta E=256(13) \mathrm{cm}^{-1}$, being similar to ab initio first excited ligand field level $\left(239-270 \mathrm{~cm}^{-1}\right)$.

\section{Summary of the magnetic properties}

As our group have mentioned about the correlation between the $\chi_{\mathrm{ax}}$ values derived from NMR analysis and the $\Delta E$ values derived from ac magnetic measurements, the similar correlation was seen in the present study. In [2] series, the oxidation reaction from [2] $]^{0}$ to $[2]^{+}$lengthens the $\tau$ values and enhances the $\Delta E$ values. Experimental $\Delta E$ values are larger than the first excited ligand field energy levels, but rather close to the values of second exited state. Although ab initio LF splitting for [2] $]^{-}$is larger than that of $[2]^{+}$, the latter complex sustains collinearity of spin directions between ground, first and second excited states, causing the larger experimental $\Delta E$ values. The experimental increases in $\Delta E$ values are consistent with the small increase in $\chi_{\mathrm{ax}}$ values by NMR analysis. In clear contrast to [2] series, the [3] shows decrease in $\Delta E$ values and $\chi_{\text {ax }}$ values by the oxidations from $[3]^{0}$ to $[3]^{2+}$. Ab initio calculations as well as empirical point-charge calculations support these experimental results. We speculate that the approach of $+3 e$ charged $\mathrm{Tb}$ (III) ions via axial compressions that are induced by ligand oxidations decrease the effect of negative charges on inner obPc ligands, thereby the axial component of the ligand field parameters around $\mathrm{Tb}(\mathrm{III})$ ions are decreased. Compared to [3], multinuclear complexes with $\mathrm{Cd}(\mathrm{II})$ ions do not show large changes in the magnetic properties via ligand oxidations owing to the approach of less positively charged $\mathrm{Cd}(\mathrm{II})$ to the $\mathrm{Tb}(\mathrm{III})$. Although $[4]^{4+}$ and $[5]^{4+}$ have two kind of $\mathrm{Tb}(\mathrm{III})$ centers (SAP and SP geometries), ac magnetic susceptibilities of them show a single peak, indicating that magnetic relaxations for SAP and SP centers are similar with each other. Ab initio calculations also support the smaller dependency of coordination geometries on the ligand field levels and the wavefunctions of the ground doublet. However, the changes of $\Delta E$ values 
are not as simple as the monotonic decrease in $\chi_{\mathrm{ax}}$ values from NMR analysis (Experimental and theoretical $\Delta E$ sometimes slightly increase and sometimes slightly decrease due to the oxidations). Although no definite reason for this discrepancy may be given, the difference in measurement conditions, that is, whether the samples are in solution (NMR measurements) or solid states (SQUID measurements), would certainly affect the relation between $\chi_{\mathrm{ax}}$ and $\Delta E$.

\section{CONCLUSION}

In this article, we performed the comprehensive study for the lanthanide-phthalocyaninato multipledecker complexes in high valence states with the help of redox measurements, solution NMR, SXRD and magnetic measurements. Electron donating $n$-butoxy chains in the present complexes enable us to isolate the high valence states of multiple-decker complexes. The solution paramagnetic NMR studies revealed the decrease in the magnetic anisotropy in the series of [3] to [5] with increasing oxidation numbers of the complexes, and the unexpected existence of $\pi$-radicals in $[4]^{2+}$ and $[5]^{2+}$. The decrease in magnetic anisotropy stems from the oxidation induced axial compression in the complexes causing the approach of intramolecular metal ions and resultant decrease in axial component of the ligand field. In addition, ESR spectra of $[4]^{2+}$ and $[5]^{2+}$ show multiple signals characteristic for a triplet biradical with zero-field spitting. Crystal structure analysis revealed that the oxidations decrease $R_{\text {obPc-obPc }}$ distances as anticipated from DFT calculations. In addition, $[4]^{4+}$ and $[5]^{4+}$ show an unusual coexistence of SAP and SP geometries, the latter being supported by solvent inclusion in the gap made by eclipsed stacking of obPc ligands. The crystal packings of oxidized complexes are affected by both the shapes of the multiple-decker units and counter ions. The shorter multiple-decker complexes ([2] and [3] series) and longer ones with planner obPc ${ }^{\circ}$ ligands $\left([4]^{0}\right.$ and $\left.[5]^{0}\right)$ construct the slipped columns structure inside which the intermolecular $\pi-\pi$ interactions are maximized, whereas longer and oxidized multiple-decker complexes $\left([4]^{2+},[4]^{4+}\right.$ and $\left.[5]^{4+}\right)$ form the $1 \mathrm{D}$ column packing along their $C_{4}$ axis because the bowl-shaped distortion on outer obPc ligands induced by the ligand oxidations prevent the intermolecular $\pi-\pi$ interactions. Especially, highly distorted $\mathrm{obPc}^{\circ}$ ligands of $+4 e$ charged species serve the space by which the counter ions $\mathrm{SbCl}_{6}{ }^{-}$are accommodated to the column packing. Ab initio calculations were used for fitting experimental data and revealed that all the multiple-decker $\mathrm{Tb}$ (III) complexes show strong axial magnetic anisotropy, as reported previously and proofed by the experimental magnetic analysis in this research. Experimental $\Delta E$ values and $a b$ initio ligand field splitting for [2] series suggest the Orbach process to be prevalent via second excited states. In [3], ligand oxidations induce the reduction of $\Delta E$ values due to a decrease in $R_{\mathrm{Tb}-\mathrm{Tb}}$, which in turn decreases the axial component of ligand field due to the approach of the neighboring $+3 e$ charges to the $\mathrm{Tb}$ (III) center. These behaviors are fully consistent with the ${ }^{1} \mathrm{H}$ NMR analysis, the ab initio calculations and empirical point-charge calculations. In contrast to [3] series, the SMM properties for [4] and [5] series, i.e. $\tau$ and $\Delta E$ values, are less dependent on the ligand oxidations. The decrease in the $R_{\mathrm{Tb}-\mathrm{Cd}}$ causes the 
approach of $+2 e$ charges to the $\mathrm{Tb}(\mathrm{III})$ ions, of which impact to the ligand-field splitting on $\mathrm{Tb}(\mathrm{III})$ is small compared to the approach of $+3 e$ charges.

From the above results, we demonstrate the versatile electrochemical, magnetic and structural properties of highly oxidized multiple-decker complexes. The ligand oxidations and the magnetic properties of the lanthanoid center couple with each other by the oxidation induced structural deformations. Durability to the multistep oxidation procedure in the present series of complexes lead to the multistep molecular switch incorporated to the molecular magnet and the molecular machines. Not only for the structural deformation, we present the strategy to synthesize the novel dicationic biradical species, where the longitudinal extension of the $\pi$-plane with electron donating groups and following a two electron oxidation results in the exceptionally low HOMO-LUMO energy gap. Importantly, the spin densities on the outer ligands become high. By extending the stacked $\pi$-structure, the distance between the monoradical spin units increases though both of them are connected by the $\pi$-plane. We believe that loss of two electrons from $\pi$-stacked molecular wires will lead to the low energy consumption information transport by reading and changing the spin states on the terminals of the wires.

\section{EXPERIMENTAL SECTION}

4-tert-Butyltoluene and phenoxathiin were purchased from Tokyo Chemical Industry and Kanto Chemical, respectively. $1 \mathrm{M}$ antimony pentachloride in $\mathrm{CH}_{2} \mathrm{Cl}_{2}$ was purchased from Sigma-Aldrich. The deuterated solvent $\left(\mathrm{CD}_{2} \mathrm{Cl}_{2}\right.$, Sigma-Aldrich, $99.9 \%$ deuteration) was dried with conventional methods ( $3 \AA$ molecular sieves) and degassed before use. All other reagents including the solvent used for column chromatography were purchased from FUJIFILM Wako Pure Chemical Corporation. All the reagents were used without further purification. The obPc ligand, $[\mathbf{2}]^{\mathbf{0}},[\mathbf{3}]^{\mathbf{0}}$ and $[\mathbf{5}]^{\mathbf{0}}$ were synthesized according to literature methods ${ }^{61,123-124}$, using reagents purchased from Wako chemicals.

\section{Synthesis for phenoxathiin hexachloroantimonate $\mathbf{O x} \cdot \mathbf{S b C l}_{\mathbf{6}}$}

To a $30 \mathrm{~mL}$ of Schenck tube, $1.0 \mathrm{~g}$ of phenoxathiin $(5.0 \mathrm{mmol})$ and $10 \mathrm{~mL}$ of dried $\mathrm{CH}_{2} \mathrm{Cl}_{2}$ were added in a flow of $\mathrm{N}_{2}$ gas. Addition of $6 \mathrm{~mL}$ of $1 \mathrm{M} \mathrm{SbCl}_{5} / \mathrm{CH}_{2} \mathrm{Cl}_{2}$ solution gave a blue solid. The solvent was removed, the blue solid was washed three times using $20 \mathrm{~mL}$ of $\mathrm{CH}_{2} \mathrm{Cl}_{2}$ and canula and was dried in vacuo (2.22 g. 83\%). Elemental analysis calculated (\%) for $\mathrm{C}_{12} \mathrm{H}_{8} \mathrm{SOSbCl}_{6}$ : C 26.95, H 1.51, N 0.00 ; found: C 27.02, H 1.60, N 0.00 .

\section{Chemical synthesis for $[2]^{+}$}

To a $10 \mathrm{~mL}$ of vial, $25 \mathrm{mg}$ of [2] $]^{0}, 8.2 \mathrm{mg}$ of phenoxathiin hexachloroantimonate and $3 \mathrm{~mL}$ of toluene were added and mixed them using ultrasonication. The vial was put in the oven and heated for $100 \mathrm{C}$ for $5 \mathrm{~h}$, followed by slow cooling down to room temperature over $12 \mathrm{~h}$ afford the crystalline $[2]^{+}(22$ $\mathrm{mg}$, ). Elemental analysis calculated (\%) for $\mathrm{C}_{128} \mathrm{H}_{160} \mathrm{~N}_{16} \mathrm{O}_{16} \mathrm{TbSbCl}_{6}$ : C 57.53, H 6.04, $\mathrm{N}$ 8.39; found: C 57.26, H 5.92, N 8.23. 


\section{Chemical synthesis for $[2]^{-}$}

The synthesis of bulk amount of $[2]^{-}$has been reported by our group. ${ }^{48}$ Single crystals suitable for SXRD measurements were prepared using the solvent diffusion method shown below. To a $2 \mathrm{~mL}$ microtube, $10 \mathrm{mg}$ of [2] $]^{0}, 100 \mu \mathrm{L}$ of chloroform, $100 \mu \mathrm{L}$ of dimethyl sulfoxide, $20 \mu \mathrm{L}$ of hydrazine monohydrate were added and mixed using ultrasonication until the color of the solution changed to blue. Slow diffusion of dimethyl sulfoxide containing $\sim 80 \mathrm{mM}$ of tetrabutylammonium bromide for $\sim 2$ weeks gave red block crystals suitable for SXRD measurements (7.6 mg, 69\%). Elemental analysis calculated (\%) for $\mathrm{C}_{144} \mathrm{H}_{196} \mathrm{~N}_{17} \mathrm{O}_{16} \mathrm{~Tb}$ : C 67.03, H 7.66, N 9.23; found: C 66.80, H 7.49, N 9.17.

\section{Chemical synthesis for $[3]^{2+}$}

$44 \mathrm{mg}$ of [3] ${ }^{0}, 16 \mathrm{mg}$ of $\mathrm{Ox} \cdot \mathrm{SbCl}_{6}$ and $2 \mathrm{~mL}$ of toluene were added to a $10 \mathrm{~mL}$ screw vial and mixed using ultrasonication. The resultant oil compound was stored at ca. $20^{\circ} \mathrm{C}$ for 1 week, affording the crystalline sample of $[3]^{2+}(46 \mathrm{mg}, 88 \%)$. Elemental analysis calculated (\%) for $\mathrm{C}_{192} \mathrm{H}_{240} \mathrm{~N}_{24} \mathrm{O}_{24} \mathrm{~Tb}_{2} \mathrm{Sb}_{2} \mathrm{Cl}_{12}$ : C 54.20, H 5.69, N 7.90; found: C 54.13, H 5.63, N 7.76.

\section{Chemical synthesis for $[4]^{2+}$}

$5.04 \mathrm{mg}$ of $[4]^{0}$ and $1.15 \mathrm{mg}$ of $\mathrm{Ox} \cdot \mathrm{SbCl}_{6}$ were mixed in ca. $0.5 \mathrm{~mL}$ of 1,1,2,2-tetrachloroethane by ultrasonication. Slow diffusion of excess 4-tert-butyltoluene (TBT) to the solution for ca. 1 week gave the crystalline sample of $[4]^{2+}(4.5 \mathrm{mg}, 78 \%)$. Elemental analysis calculated (\%) for $[4]^{2+}$ containing 0.5 equivalent of TCE $\mathrm{C}_{257} \mathrm{H}_{321} \mathrm{~N}_{32} \mathrm{O}_{32} \mathrm{~Tb}_{2} \mathrm{CdSb}_{2} \mathrm{Cl}_{14}$ : C 55.71, H 5.84, $\mathrm{N}$ 8.09; found: $\mathrm{C} 55.63, \mathrm{H}$ 5.81, N 8.01.

\section{Chemical synthesis for $[4]^{4+}$}

$5.78 \mathrm{mg}$ of $[4]^{0}$ and $2.89 \mathrm{mg}$ of $\mathrm{Ox} / \mathrm{SbCl}_{6}$ were mixed in ca. $0.5 \mathrm{~mL}$ of TCE by ultrasonication. Slow diffusion of excess TBT to the solution during ca. 1 week gave the crystalline sample of $[4]^{4+}(6.2 \mathrm{mg}$, 84\%). Elemental analysis calculated (\%) for $[4]^{4+}$ containing 0.2 equivalent of TBT $\mathrm{C}_{262.6} \mathrm{H}_{329.6} \mathrm{~N}_{32} \mathrm{O}_{32} \mathrm{~Tb}_{2} \mathrm{CdSb}_{4} \mathrm{Cl}_{24}$ : C 50.75, H 5.35, N 7.21; found: C 50.53, H 5.16, N 6.91.

\section{Chemical synthesis for $[5]^{4+}$}

$5.77 \mathrm{mg}$ of $[5]^{0}$ and $2.29 \mathrm{mg}$ of $\mathrm{Ox} \cdot \mathrm{SbCl}_{6}$ were mixed in ca. $0.5 \mathrm{~mL}$ of TCE by ultrasonication. Slow diffusion of excess benzene to the solution over ca. 1 week gave the crystalline sample of $[5]^{4+}(5.8$ $\mathrm{mg}, 82 \%)$. This sample spontaneously melts after drying because of the remaining 1,1,2,2-TCE. For collection of samples, we replaced the mother liquor with pure benzene by centrifuging and exchanging the solvent. Elemental analysis calculated (\%) for $\mathrm{C}_{320} \mathrm{H}_{400} \mathrm{~N}_{40} \mathrm{O}_{40} \mathrm{~Tb}_{2} \mathrm{Cd}_{2} \mathrm{Sb}_{4} \mathrm{Cl}_{24}: \mathrm{C} 52.45$, H 5.50, N 7.65; found: C 52.26, H 5.44, N 7.53.

\section{Measurement and Calculation Details}

Bulk electrolysis for multiple-decker complexes were conducted using Metrohm Autolab PGSTAT101 and ALS Model $2325 \mathrm{Bi}$-potentiostat with the $\mathrm{Ag} / \mathrm{Ag}^{+}$reference eletrode, platinum working electrode and platinum reference electrode separated by vycor bridge udner $\mathrm{N}_{2}$ atmosphere. UV-Vis-NIR absorption spectra are aquired using Agilent Cary 5000 UV-Vis-NIR and SHIMADZU UV-3100PC in 
a quartz cell with a path length of $1 \mathrm{~mm}$ at room temperature. For electrochemical UV-Vis-NIR measurements, the reaction product solution of the bulk electrolysis was transferred to a sealed quarts cell using Hamilton syringe. NMR spectra were recorded at a magnetic field of $9.4 \mathrm{~T}$ with a Bruker Avance II instrument equipped with a BBO probehead and at a magnetic field of $14.09 \mathrm{~T}$ with a Bruker Avance III instrument equipped with a cryo-probehead. SXRD measurements were performed using Rigaku Varimax with Saturn724+ with CrysAlisPro $3.9^{125}$ and Rigaku VariMax with RAPID II with RAPID AUTO. Initial structures were obtained using SHELXT (2014/7) and refined with SHELXL $(2014 / 7){ }^{126}$ combined with Yadokari-XG. ${ }^{127}$ Powder X-ray diffraction pattern was acquired by Rigaku RINT-2000 with a glass capillary. Magnetic measurements were performed on Quantum Design SQUID magnetometers MPMS2, MPMS-XL and MPMS3.Details about the DFT and Ab initio ligand field calculations are given in Supporting Information.

\section{AUTHOR INFORMATION}

\section{Corresponding Authors}

Y.H.: horiiy17@chem.sci.osaka-u.ac.jp

M.D.: marko.damjanovic@kit.edu

K.K.: kkatoh@m.tohoku.ac.jp

M.E.: markus.enders@uni-heidelberg.de

J.V.: vecianaj@icmab.es

M.Y.: yamasita@agnus.chem.tohoku.ac.jp

\section{Notes}

First two authors (Y.H. and M.D.) contributed equally to this work.

\section{ACKNOWLEDGEMENTS}

We express our gratitude for the following research funds: a $\mathrm{PhD}$ scholarship for M.D. from the Beilstein-Institut zur Förderung der Chemischen Wissenschaften, the German-Japanese University Consortium (HeKKSaGOn) for travel supports (all authors). We are grateful for the computational resources provided by the bwForCluster JUSTUS, funded by the State of Baden-Württemberg, Germany, and the German Research Foundation (DFG) through grant No. INST 40/467-1 FUGG. This work was financially supported by Japan Society for the Promotion of Science (JSPS) KAKENHI Grant Numbers JP14J02656 (Y.H.), JP20225003 (M.Y.), JP15K05467 (K.K.), JP24750119 (K.K.), JP17K19102 (M.N.), 18K14242 (Y.H.), 19K05401(Y.K), Tohoku University Molecule \&Material Synthesis Platform in Nanotechnology Platform Project, and CREST, JST JPMJCR12L3 (M.Y.), Japan. This work was also funded by European Union (ERC StG 2012-306826 e-GAMES), Networking Research Center on Bioengineering, Biomaterials, and Nanomedicine (CIBER-BBN), the Spanish Ministry of Economy and Competitiveness (projects FANCY CTQ2016-80030-R, MOTHER MAT2016-80826-R and the "Severo Ochoa" Programme for Centers of Excellence in R\&D, SEV- 
2015-0496) and by Generalitat de Catalunya (2017SGR918). We thank Kazushi Kawamura for IR spectroscopic measurements, Ken-ichi Iijima and Tomomi Hirai for CHN elemental analyses, Dr. Norihisa Hoshino and Prof. Dr. Tomoyuki Akutagawa for EPR measurements, Prof. Dr. Motohiro Nakano for SQUID measurements, Dr. Tetsuko Nakaniwa and Prof. Dr. Genji Kurisu for setup of SXRD instruments, Dr Takefumi Yoshida for X-ray structural analyses. This paper is Contribution No. 62 from the Research Center for Thermal and Entropic Science. M. Y. thanks for support from the 111 Project (B18030) from China. The scientific grants R-143-000-A80-114, R-143-000-A65-133 from the National University of Singapore are gratefully acknowledged.

\section{REFERENCES}

1. Roznyatovskiy, V. V.; Lee, C.-H.; Sessler, J. L., $\pi$-Extended isomeric and expanded porphyrins. Chem. Soc. Rev. 2013, 42 (5), 1921-1933.

2. Yano, Y.; Mitoma, N.; Matsushima, K.; Wang, F.; Matsui, K.; Takakura, A.; Miyauchi, Y.; Ito, H.; Itami, K., Living annulative $\pi$-extension polymerization for graphene nanoribbon synthesis. Nature 2019.

3. Cheng, Y.-J.; Yang, S.-H.; Hsu, C.-S., Synthesis of Conjugated Polymers for Organic Solar Cell Applications. Chem. Rev. 2009, 109 (11), 5868-5923.

4. Grimsdale, A. C.; Leok Chan, K.; Martin, R. E.; Jokisz, P. G.; Holmes, A. B., Synthesis of LightEmitting Conjugated Polymers for Applications in Electroluminescent Devices. Chem. Rev. 2009, 109 (3), 897-1091.

5. Morita, Y.; Nishida, S.; Murata, T.; Moriguchi, M.; Ueda, A.; Satoh, M.; Arifuku, K.; Sato, K.; Takui, T., Organic tailored batteries materials using stable open-shell molecules with degenerate frontier orbitals. Nat. Mater. 2011, 10, 947.

6. Wang, C.; Xu, Y.; Fang, Y.; Zhou, M.; Liang, L.; Singh, S.; Zhao, H.; Schober, A.; Lei, Y., Extended $\pi$-Conjugated System for Fast-Charge and -Discharge Sodium-Ion Batteries. J. Am. Chem. Soc. 2015, 137 (8), 3124-3130.

7. Hunter, C. A.; Sanders, J. K. M., The nature of $\pi-\pi$ interactions. J. Am. Chem. Soc. 1990, 112 (14), 5525-5534.

8. Narita, A.; Wang, X.-Y.; Feng, X.; Müllen, K., New advances in nanographene chemistry. Chem. Soc. Rev. 2015, 44 (18), 6616-6643.

9. Tsuda, A.; Osuka, A., Fully Conjugated Porphyrin Tapes with Electronic Absorption Bands That Reach into Infrared. Science 2001, 293 (5527), 79-82.

10. Kobayashi, A.; Fujiwara, E.; Kobayashi, H., Single-Component Molecular Metals with Extended-TTF Dithiolate Ligands. Chem. Rev. 2004, 104 (11), 5243-5264.

11. Ferraris, J.; Cowan, D. O.; Walatka, V.; Perlstein, J. H., Electron transfer in a new highly conducting donor-acceptor complex. J. Am. Chem. Soc. 1973, 95 (3), 948-949. 

metals and superconductors derived from metal complexes of 1,3-dithiol-2-thione-4,5-dithiolate (dmit). Coord. Chem. Rev. 1991, 110 (1), 115-160.

13. Petersen, J. L.; Schramm, C. S.; Stojakovic, D. R.; Hoffman, B. M.; Marks, T. J., A new class of highly conductive molecular solids: the partially oxidized phthalocyanines. J. Am. Chem. Soc. 1977, 99 (1), 286-288.

14. Davison, J. B.; Wynne, K. J., Silicon Phthalocyanine-Siloxane Polymers: Synthesis and ${ }^{1} \mathrm{H}$ Nuclear Magnetic Resonance Study. Macromolecules 1978, 11 (1), 186-191.

15. Hanack, M.; Hirsch, A.; Lehmann, H., Soluble, Oligomeric Bridged Phthalocyaninatoiron(II) Complexes. Angew. Chem. Int. Ed. 1990, 29 (12), 1467-1468.

16. Venkata Rao, K.; Miyajima, D.; Nihonyanagi, A.; Aida, T., Thermally bisignate supramolecular polymerization. Nat. Chem. 2017, 9, 1133.

17. Janson, T. R.; Kane, A. R.; Sullivan, J. F.; Knox, K.; Kenney, M. E., Ring-current effect of the phthalocyanine ring. J. Am. Chem. Soc. 1969, 91 (19), 5210-5214.

18. DeWulf, D. W.; Leland, J. K.; Wheeler, B. L.; Bard, A. J.; Batzel, D. A.; Dininny, D. R.; Kenney, M., Isolation, spectroscopic properties, and electrochemical properties of two oligomeric silicon phthalocyanines. Inorg. Chem. 1987, 26 (2), 266-270.

19. Anderson, A. B.; Gordon, T. L.; Kenney, M. E., Electronic and redox properties of stacked-ring silicon phthalocyanines from molecular orbital theory. J. Am. Chem. Soc. 1985, 107 (1), 192-195.

20. Fukuda, T.; Biyajima, T.; Kobayashi, N., A Discrete Quadruple-Decker Phthalocyanine. J. Am. Chem. Soc. 2010, 132 (18), 6278-6279.

21. Wang, H.; Kobayashi, N.; Jiang, J., New Sandwich-Type Phthalocyaninato-Metal QuintupleDecker Complexes. Chem. Eur. J. 2012, 18 (4), 1047-1049.

22. Wang, H.; Qi, D.; Xie, Z.; Cao, W.; Wang, K.; Shang, H.; Jiang, J., A sandwich-type phthalocyaninato metal sextuple-decker complex: synthesis and NLO properties. Chem. Commun. 2013, 49 (9), 889-891.

23. Kirin, I. S.; Moskalev, P. N.; Makashev, Y. A., Formation of unusual phthalocyanines of the rareearth elements. Russ. J. Inorg. Chem. 1965, 10 (8), 1065-1066.

24. Kirin, I.; Moskalev, P.; Maskashev, Y., New complexes of phthalocyanine with rare-earth elements. Russ. J. Inorg. Chem 1967, 12 (3), 369.

25. Kirin, I. S.; Moskalev, P. N.; Ivannikova, N. V., Preparation and properties of neodymium phthalocyaniene. Russ. J. Inorg. Chem 1967, 12 (4), 497-498.

26. Buchler, J. W.; De Cian, A.; Fischer, J.; Kihn-Botulinski, M.; Paulus, H.; Weiss, R., Metal complexes with tetrapyrrole ligands. 40. Cerium(IV) bis(octaethylporphyrinate) and dicerium(III) tris(octaethylporphyrinate): Parents of a new family of lanthanoid double-decker and triple-decker molecules. J. Am. Chem. Soc. 1986, 108 (13), 3652-3659. 
27. Chabach, D.; De Cian, A.; Fischer, J.; Weiss, R.; Bibout, M. E. M., Mixed-Metal Triple-Decker Sandwich Complexes with the Porphyrin/Phthalocyanine/Porphyrin Ligand System. Angew. Chem. Int. Ed. 1996, 35 (8), 898-899.

28. Chabach, D.; Tahiri, M.; De Cian, A.; Fischer, J.; Weiss, R.; Bibout, M. E. M., Tervalent-Metal Porphyrin-Phthalocyanine Heteroleptic Sandwich-Type Complexes. Synthesis, Structure, and Spectroscopic Characterization of Their Neutral, Singly-Oxidized, and Singly-Reduced States. J. Am. Chem. Soc. 1995, 117 (33), 8548-8556.

29. Ishikawa, N.; Ohno, O.; Kaizu, Y., Hole delocalization in naphthalocyaninatophthalocyaninatolutetium (III). Chem. Phys. Lett. 1991, 180 (1), 51-56.

30. Ishikawa, N.; Ohno, O.; Kaizu, Y.; Kobayashi, H., Localized orbital study on the electronic structure of phthalocyanine dimers. J. Phys. Chem. C 1992, 96 (22), 8832-8839.

31. Ishikawa, N.; Ohno, O.; Kaizu, Y., Electronic states of bis(phthalocyaninato)lutetium radical and its related compounds: the application of localized orbital basis set to open-shell phthalocyanine dimers. $J$. Phys. Chem. C 1993, 97 (5), 1004-1010.

32. Ishikawa, N.; Kaizu, Y., Axially polarized NIR absorption bands in electron- deficient lanthanide phthalocyanine dimers and trimers. J. Porphyr. Phthalocyanines 1999, 3 (6 - 7), 514-521.

33. Ishikawa, N., Electronic structures and spectral properties of -double- and triple-decker phthalocyanine complexes -in a localized molecular orbital view. J. Porphyr. Phthalocyanines 2001, 5 (1), 87-101.

34. Ishikawa, N.; Iino, T.; Kaizu, Y., Interaction between f-Electronic Systems in Dinuclear Lanthanide Complexes with Phthalocyanines. J. Am. Chem. Soc. 2002, 124 (38), 11440-11447.

35. Ishikawa, N.; Iino, T.; Kaizu, Y., Determination of Ligand-Field Parameters and f-Electronic Structures of Hetero-Dinuclear Phthalocyanine Complexes with a Diamagnetic Yttrium(III) and a Paramagnetic Trivalent Lanthanide Ion. J. Phys. Chem. A 2002, 106 (41), 9543-9550.

36. Ishikawa, N., Simultaneous Determination of Ligand-Field Parameters of Isostructural Lanthanide Complexes by Multidimensional Optimization. J. Phys. Chem. A 2003, 107 (30), 5831-5835.

37. Ishikawa, N.; Iino, T.; Kaizu, Y., Study of ${ }^{1} H$ NMR Spectra of Dinuclear Complexes of Heavy Lanthanides with Phthalocyanines Based on Separation of the Effects of Two Paramagnetic Centers. J. Phys. Chem. A 2003, 107 (39), 7879-7884.

38. Ruan, C.-y.; Mastryukov, V.; Fink, M., Electron diffraction studies of metal phthalocyanines, MPc, where M=Sn, Mg, and Zn (reinvestigation). J. Chem. Phys. 1999, 111 (7), 3035-3041.

39. Koike, N.; Uekusa, H.; Ohashi, Y.; Harnoode, C.; Kitamura, F.; Ohsaka, T.; Tokuda, K., Relationship between the Skew Angle and Interplanar Distance in Four Bis(phthalocyaninato)lanthanide(III) Tetrabutylammonium Salts ([NBun $\left.{ }^{4}\right]\left[\mathrm{Ln}^{\mathrm{IIIP}} \mathrm{c}_{2}\right]$; $\mathrm{Ln}=\mathrm{Nd}, \mathrm{Gd}, \mathrm{Ho}, \mathrm{Lu}$ ). Inorg. Chem. 1996, 35 (20), 5798-5804.

40. Ishikawa, N.; Sugita, M.; Ishikawa, T.; Koshihara, S. Y.; Kaizu, Y., Lanthanide double-decker 
complexes functioning as magnets at the single-molecular level. J. Am. Chem. Soc. 2003, 125 (29), 86945 .

41. Ishikawa, N.; Sugita, M.; Okubo, T.; Tanaka, N.; Iino, T.; Kaizu, Y., Determination of ligandfield parameters and f-electronic structures of double-decker bis(phthalocyaninato)lanthanide complexes. Inorg. Chem. 2003, 42 (7), 2440-6.

42. Passard, M.; Blanc, J. P.; Maleysson, C., Gaseous oxidation and compensating reduction of lutetium bis-phthalocyanine and lutetium phthalo-naphthalocyanine films. Thin Solid Films 1995, 271 (1), $8-14$.

43. Katoh, K.; Yoshida, Y.; Yamashita, M.; Miyasaka, H.; Breedlove, B. K.; Kajiwara, T.; Takaishi, S.; Ishikawa, N.; Isshiki, H.; Zhang, Y. F.; Komeda, T.; Yamagishi, M.; Takeya, J., Direct Observation of Lanthanide(III)-Phthalocyanine Molecules on $\mathrm{Au}(111)$ by Using Scanning Tunneling Microscopy and Scanning Tunneling Spectroscopy and Thin-Film Field-Effect Transistor Properties of Tb(III)- and Dy(III)Phthalocyanine Molecules. J. Am. Chem. Soc. 2009, 131 (29), 9967-9976.

44. Katoh, K.; Yamamoto, K.; Kajiwara, T.; Takeya, J.; Breedlove, B. K.; Yamashita, M., Magnetic properties of lanthanoid(III) phthalocyaninato triple-decker complexes in an external magnetic field and electronic transport properties for molecular spintronics. J. Phys. Conf. Ser. 2011, 303 (1), 012035.

45. Sugasaki, A.; Ikeda, M.; Takeuchi, M.; Robertson, A.; Shinkai, S., Efficient chirality transcription utilizing a cerium(IV) double decker porphyrin: a prototype for development of a molecular memory system. J. Chem. Soc. Perkin 1 1999, (22), 3259-3264.

46. Ogi, S.; Ikeda, T.; Wakabayashi, R.; Shinkai, S.; Takeuchi, M., A Bevel-Gear-Shaped Rotor Bearing a Double-Decker Porphyrin Complex. Chem. Eur. J. 2010, 16 (28), 8285-8290.

47. Shibata, M.; Tanaka, S.; Ikeda, T.; Shinkai, S.; Kaneko, K.; Ogi, S.; Takeuchi, M., StimuliResponsive Folding and Unfolding of a Polymer Bearing Multiple Cerium(IV) Bis(porphyrinate) Joints: Mechano-imitation of the Action of a Folding Ruler. Angew. Chem. Int. Ed. 2013, 52 (1), 397-400.

48. Damjanovic, M.; Morita, T.; Katoh, K.; Yamashita, M.; Enders, M., Ligand $\pi$-radical interaction with f-shell unpaired electrons in phthalocyaninato-lanthanoid single-molecule magnets: a solution NMR spectroscopic and DFT study. Chem. Eur. J. 2015, 21 (41), 14421-32.

49. Yamashita, K.-i.; Yamanaka, T.; Sakata, N.; Ogawa, T., Redox-Driven Symmetry Change for Terbium(III) Bis(porphyrinato) Double-Decker Complexes by the Azimuthal Rotation of the Porphyrin Macrocycles. Chem. Asian. J. 2018, 13 (13), 1692-1698.

50. Caneschi, A.; Gatteschi, D.; Sessoli, R.; Barra, A. L.; Brunel, L. C.; Guillot, M., Alternating current susceptibility, high field magnetization, and millimeter band EPR evidence for a ground $S=10$ state in $\left[\mathrm{Mn}_{12} \mathrm{O}_{12}\left(\mathrm{CH}_{3} \mathrm{COO}\right)_{16}\left(\mathrm{H}_{2} \mathrm{O}\right)_{4}\right] \cdot 2 \mathrm{CH}_{3} \mathrm{COOH} \cdot 4 \mathrm{H}_{2} \mathrm{O}$. J. Am. Chem. Soc. 1991, 113 (15), 5873-5874.

51. Komeda, T.; Isshiki, H.; Liu, J.; Zhang, Y.-F.; Lorente, N.; Katoh, K.; Breedlove, B. K.; Yamashita, M., Observation and electric current control of a local spin in a single-molecule magnet. Nat. Commun. 2011, 2, 217. 
52. Moreno-Pineda, E.; Klyatskaya, S.; Du, P.; Damjanovic, M.; Taran, G.; Wernsdorfer, W.; Ruben, M., Observation of Cooperative Electronic Quantum Tunneling: Increasing Accessible Nuclear States in a Molecular Qudit. Inorg. Chem. 2018, 57 (16), 9873-9879.

53. Katoh, K.; Aizawa, Y.; Morita, T.; Breedlove, B. K.; Yamashita, M., Elucidation of Dual Magnetic Relaxation Processes in Dinuclear Dysprosium(III) Phthalocyaninato Triple-Decker SingleMolecule Magnets Depending on the Octacoordination Geometry. Chem. Eur. J. 2017, 23 (61), 1537715386.

54. Morita, T.; Katoh, K.; Breedlove, B. K.; Yamashita, M., Controlling the dipole-dipole interactions between terbium(III) phthalocyaninato triple-decker moieties through spatial control using a fused phthalocyaninato ligand. Inorg. Chem. 2013, 52 (23), 13555-61.

55. Bogani, L.; Wernsdorfer, W., Molecular spintronics using single-molecule magnets. Nat. Mater. 2008, 7, 179 .

56. Vincent, R.; Klyatskaya, S.; Ruben, M.; Wernsdorfer, W.; Balestro, F., Electronic read-out of a single nuclear spin using a molecular spin transistor. Nature 2012, 488, 357.

57. Urdampilleta, M.; Klyatskaya, S.; Cleuziou, J. P.; Ruben, M.; Wernsdorfer, W., Supramolecular spin valves. Nat. Mater. 2011, 10, 502.

58. Ganzhorn, M.; Klyatskaya, S.; Ruben, M.; Wernsdorfer, W., Strong spin-phonon coupling between a single-molecule magnet and a carbon nanotube nanoelectromechanical system. Nat. Nanotechnol. 2013, 8,165 .

59. Kyatskaya, S.; Galán Mascarós, J. R.; Bogani, L.; Hennrich, F.; Kappes, M.; Wernsdorfer, W.; Ruben, M., Anchoring of Rare-Earth-Based Single-Molecule Magnets on Single-Walled Carbon Nanotubes. J. Am. Chem. Soc. 2009, 131 (42), 15143-15151.

60. Kizaki, K.; Ozawa, H.; Kobayashi, T.; Matsuoka, R.; Sakaguchi, Y.; Fuyuhiro, A.; Fukuda, T.; Ishikawa, N., Coupling between the photo-excited cyclic pi system and the $4 \mathrm{f}$ electronic system in a lanthanide single molecule magnet. Chem. Commun. 2017, 53 (45), 6168-6171.

61. Horii, Y.; Katoh, K.; Yasuda, N.; Breedlove, B. K.; Yamashita, M., Effects of f-f Interactions on the Single-Molecule Magnet Properties of Terbium(III)-Phthalocyaninato Quintuple-Decker Complexes. Inorg. Chem. 2015, 54 (7), 3297-3305.

62. Tao, J.; Perdew, J. P.; Staroverov, V. N.; Scuseria, G. E., Climbing the Density Functional Ladder: Nonempirical Meta Generalized Gradient Approximation Designed for Molecules and Solids. Phys. Rev. Lett. 2003, 91 (14), 146401.

63. Weigend, F.; Ahlrichs, R., Balanced basis sets of split valence, triple zeta valence and quadruple zeta valence quality for $\mathrm{H}$ to Rn: Design and assessment of accuracy. Phys. Chem. Chem. Phys. 2005, 7 (18), 3297-3305.

64. Weigend, F., Accurate Coulomb-fitting basis sets for H to Rn. Phys. Chem. Chem. Phys. 2006, 8 (9), 1057-1065. 
65. Miertuš, S.; Scrocco, E.; Tomasi, J., Electrostatic interaction of a solute with a continuum. A direct utilizaion of Ab initio molecular potentials for the prevision of solvent effects. Chem. Phys. 1981, 55 (1), 117-129.

66. Markovitsi, D.; Tran-Thi, T.-H.; Even, R.; Simon, J., Near infrared absorption spectra of lanthanide bis-phthalocyanines. Chem. Phys. Lett. 1987, 137 (2), 107-112.

67. Fukuda, T.; Hata, K.; Ishikawa, N., Observation of Exceptionally Low-Lying $\pi-\pi^{*}$ Excited States in Oxidized Forms of Quadruple-Decker Phthalocyanine Complexes. J. Am. Chem. Soc. 2012, 134 (36), 14698-14701.

68. Bertini, I.; Luchinat, C., Chapter 2 The hyperfine shift. Coord. Chem. Rev. 1996, 150 (0), 29-75.

69. Enders, M., Assigning and Understanding NMR Shifts of Paramagnetic Metal Complexes. In Modeling of Molecular Properties, Comba, P., Ed. Wiley-VCH Verlag GmbH \& Co. KGaA: Weinheim, Germany, 2011.

70. Mao, J.; Zhang, Y.; Oldfield, E., Nuclear magnetic resonance shifts in paramagnetic metalloporphyrins and metalloproteins. J. Am. Chem. Soc. 2002, 124 (46), 13911-20.

71. Knorr, R.; Hauer, H.; Weiss, A.; Polzer, H.; Ruf, F.; Low, P.; Dvortsak, P.; Bohrer, P., Unpaired spin densities from NMR shifts and magnetic anisotropies of pseudotetrahedral cobalt(II) and nickel(II) vinamidine bis(chelates). Inorg. Chem. 2007, 46 (20), 8379-90.

72. Kleingardner, J. G.; Bowman, S. E.; Bren, K. L., The influence of heme ruffling on spin densities in ferricytochromes c probed by heme core ${ }^{13}$ C NMR. Inorg. Chem. 2013, 52 (22), 12933-46.

73. Liimatainen, H.; Pennanen, T. O.; Vaara, J., 1H chemical shifts in nonaxial, paramagnetic chromium(III) complexes - Application of novel pNMR shift theory. Can. J. Chem. 2009, 87 (7), 954964.

74. Pennanen, T. O.; Vaara, J., Nuclear magnetic resonance chemical shift in an arbitrary electronic spin state. Phys. Rev. Lett. 2008, 100 (13), 133002.

75. La Mar, G. N., NMR of Paramagnetic Molecules - Principles and Applications. Academic Press, INC.: New York and London, 1973.

76. Bleaney, B., Nuclear magnetic resonance shifts in solution due to lanthanide ions. J Magn Reson 1972, $8(1), 91-100$.

77. Ouali, N.; Rivera, J. P.; Morgantini, P. Y.; Weber, J.; Piguet, C., The solution structure of homotrimetallic lanthanide helicates investigated with novel model-free multi-centre paramagnetic NMR methods. Dalton Trans. 2003, (7), 1251-1263.

78. Damjanovic, M.; Katoh, K.; Yamashita, M.; Enders, M., Combined NMR analysis of huge residual dipolar couplings and pseudocontact shifts in terbium(III)-phthalocyaninato single molecule magnets. J. Am. Chem. Soc. 2013, 135 (38), 14349-58.

79. Morita, T.; Damjanović, M.; Katoh, K.; Kitagawa, Y.; Yasuda, N.; Lan, Y.; Wernsdorfer, W.; Breedlove, B. K.; Enders, M.; Yamashita, M., Comparison of the Magnetic Anisotropy and Spin Relaxation 
Phenomenon of Dinuclear Terbium(III) Phthalocyaninato Single-Molecule Magnets Using the Geometric Spin Arrangement. J. Am. Chem. Soc. 2018, 140 (8), 2995-3007.

80. Suturina, E. A.; Haussinger, D.; Zimmermann, K.; Garbuio, L.; Yulikov, M.; Jeschke, G.; Kuprov, I., Model-free extraction of spin label position distributions from pseudocontact shift data. Chem Sci 2017, 8 (4), 2751-2757.

81. Charnock, G. T.; Kuprov, I., A partial differential equation for pseudocontact shift. Phys. Chem. Chem. Phys. 2014, 16 (37), 20184-9.

82. Hogben, H. J.; Krzystyniak, M.; Charnock, G. T.; Hore, P. J.; Kuprov, I., Spinach--a software library for simulation of spin dynamics in large spin systems. J. Magn. Reson. 2011, 208 (2), 179-94.

83. Becke, A. D., Density - functional thermochemistry. III. The role of exact exchange. J. Chem. Phys. 1993, 98 (7), 5648-5652.

84. Lee, C.; Yang, W.; Parr, R. G., Development of the Colle-Salvetti correlation-energy formula into a functional of the electron density. Phys. Rev. B 1988, 37 (2), 785-789.

85. Ditchfield, R.; Hehre, W. J.; Pople, J. A., Self - Consistent Molecular - Orbital Methods. IX. An Extended Gaussian - Type Basis for Molecular - Orbital Studies of Organic Molecules. J. Chem. Phys. 1971, 54 (2), 724-728.

86. Hariharan, P. C.; Pople, J. A., The influence of polarization functions on molecular orbital hydrogenation energies. Theor. Chem. Acc. 1973, 28 (3), 213-222.

87. Hehre, W. J.; Ditchfield, R.; Pople, J. A., Self-Consistent Molecular Orbital Methods. XII. Further Extensions of Gaussian-Type Basis Sets for Use in Molecular Orbital Studies of Organic Molecules. J. Chem. Phys. 1972, 56 (5), 2257-2261.

88. Andrae, D.; Häußermann, U.; Dolg, M.; Stoll, H.; Preuß, H., Energy-adjustedab initio pseudopotentials for the second and third row transition elements. Theor. Chem. Acc. 1990, 77 (2), 123-141. 89. Dolg, M.; Stoll, H.; Preuss, H.; Pitzer, R. M., Relativistic and correlation effects for element 105 (hahnium, Ha): a comparative study of $\mathrm{M}$ and $\mathrm{MO}(\mathrm{M}=\mathrm{Nb}, \mathrm{Ta}, \mathrm{Ha})$ using energy-adjusted ab initio pseudopotentials. J. Phys. Chem. C 1993, 97 (22), 5852-5859.

90. Ishikawa, N.; Kaizu, Y., Cation- and Solvent-Induced Formation of Supramolecular Structures Composed of Crown-Ether Substituted Double-Decker Phthalocyanine Radicals. J. Phys. Chem. A 2000, 104 (44), 10009-10016.

91. Wang, K.; Qi, D.; Wang, H.; Cao, W.; Li, W.; Liu, T.; Duan, C.; Jiang, J., Binuclear Phthalocyanine-Based Sandwich-Type Rare Earth Complexes: Unprecedented Two $\pi$-Bridged BiradicalMetal Integrated SMMs. Chem. Eur. J. 2013, 19 (34), 11162-11166.

92. Lee, S.; Yamashita, K.-i.; Sakata, N.; Hirao, Y.; Ogawa, K.; Ogawa, T., Stable Singlet Biradicals of Rare-Earth-Fused Diporphyrin-Triple-Decker Complexes with Low Energy Gaps and Multi-Redox States. Chem. Eur. J. 2019, 25 (13), 3240-3243.

93. Ishikawa, N.; Kaizu, Y., Biradical state in phthalocyanine $(2+2)$ tetramer composed of two bis 
(phthalocyaninato) lutetium radicals. Chem. Phys. Lett. 1993, 203 (5), 472-476.

94. Ishikawa, N.; Kaizu, Y., Synthetic, spectroscopic and theoretical study of novel supramolecular structures composed of lanthanide phthalocyanine double-decker complexes. Coord. Chem. Rev. 2002, 226 (1), 93-101.

95. Takamatsu, S.; Ishikawa, N., A theoretical study of a drastic structural change of bis(phthalocyaninato)lanthanide by ligand oxidation: Towards control of ligand field strength and magnetism of single-lanthanide-ionic single molecule magnet. Polyhedron 2007, 26 (9), 1859-1862.

96. Takamatsu, S.; Ishikawa, T.; Koshihara, S.-y.; Ishikawa, N., Significant Increase of the Barrier Energy for Magnetization Reversal of a Single-4f-Ionic Single-Molecule Magnet by a Longitudinal Contraction of the Coordination Space. Inorg. Chem. 2007, 46 (18), 7250-7252.

97. Ishikawa, N.; Mizuno, Y.; Takamatsu, S.; Ishikawa, T.; Koshihara, S.-y., Effects of Chemically Induced Contraction of a Coordination Polyhedron on the Dynamical Magnetism of Bis(phthalocyaninato)disprosium, a Single-4f-Ionic Single-Molecule Magnet with a Kramers Ground State. Inorg. Chem. 2008, 47 (22), 10217-10219.

98. Tashiro, K.; Konishi, K.; Aida, T., Metal Bisporphyrinate Double-Decker Complexes as RedoxResponsive Rotating Modules. Studies on Ligand Rotation Activities of the Reduced and Oxidized Forms Using Chirality as a Probe. J. Am. Chem. Soc. 2000, 122 (33), 7921-7926.

99. Frisch, M. J.; Trucks, G. W.; Schlegel, H. B.; Scuseria, G. E.; Robb, M. A.; Cheeseman, J. R.; Scalmani, G.; Barone, V.; Mennucci, B.; Petersson, G. A.; Nakatsuji, H.; Caricato, M.; Li, X.; Hratchian, H. P.; Izmaylov, A. F.; Bloino, J.; Zheng, G.; Sonnenberg, J. L.; Hada, M.; Ehara, M.; Toyota, K.; Fukuda, R.; Hasegawa, J.; Ishida, M.; Nakajima, T.; Honda, Y.; Kitao, O.; Nakai, H.; Vreven, T.; Montgomery Jr., J. A.; Peralta, J. E.; Ogliaro, F.; Bearpark, M. J.; Heyd, J.; Brothers, E. N.; Kudin, K. N.; Staroverov, V. N.; Kobayashi, R.; Normand, J.; Raghavachari, K.; Rendell, A. P.; Burant, J. C.; Iyengar, S. S.; Tomasi, J.; Cossi, M.; Rega, N.; Millam, N. J.; Klene, M.; Knox, J. E.; Cross, J. B.; Bakken, V.; Adamo, C.; Jaramillo, J.; Gomperts, R.; Stratmann, R. E.; Yazyev, O.; Austin, A. J.; Cammi, R.; Pomelli, C.; Ochterski, J. W.; Martin, R. L.; Morokuma, K.; Zakrzewski, V. G.; Voth, G. A.; Salvador, P.; Dannenberg, J. J.; Dapprich, S.; Daniels, A. D.; Farkas, Ö.; Foresman, J. B.; Ortiz, J. V.; Cioslowski, J.; Fox, D. J. Gaussian 09, Gaussian, Inc.: Wallingford, CT, USA, 2009.

100. Katoh, K.; Yamashita, S.; Yasuda, N.; Kitagawa, Y.; Breedlove, B. K.; Nakazawa, Y.; Yamashit, M., Control of the Spin Dynamics of Single - Molecule Magnets using a Quasi One - Dimensional Arrangement. Angew. Chem. Int. Ed. 2018.

101. Katoh, K.; Breedlove, B. K.; Yamashita, M., Symmetry of octa-coordination environment has a substantial influence on dinuclear TbIII triple-decker single-molecule magnets. Chem. Sci. 2016, 7 (7), 4329-4340.

102. Ungur, L.; Chibotaru, L. F., Ab Initio Crystal Field for Lanthanides. Chem. Eur. J. 2017, 23 (15), 3708-3718. 
103. Notes, The Art and Science of Growing Crystals. 1962.

104. Evans, G. J., Influence of external fields on nucleation and crystal growth. Crystal growth of $n$ octylbiphenyl from solution in the presence of magnetic and electromagnetic fields. J. Chem. Soc. Faraday Trans. 1985, 81 (3), 673-678.

105. Kao, A.; Pericleous, K.; Patel, M. K.; Voller, V., Effects of magnetic fields on crystal growth. Int. J. Cast. Metal. Res. 2009, 22 (1-4), 147-150.

106. Koizumi, H.; Uda, S.; Fujiwara, K.; Tachibana, M.; Kojima, K.; Nozawa, J., Technique for HighQuality Protein Crystal Growth by Control of Subgrain Formation under an External Electric Field. Crystals 2016, 6 (8), 95.

107. Potticary, J.; Terry, L. R.; Bell, C.; Papanikolopoulos, A. N.; Christianen, P. C.; Engelkamp, H.; Collins, A. M.; Fontanesi, C.; Kociok-Kohn, G.; Crampin, S.; Da Como, E.; Hall, S. R., An unforeseen polymorph of coronene by the application of magnetic fields during crystal growth. Nat. Commun. 2016, 7,11555 .

108. Yurov, V. M.; Guchenko, S. A.; Gyngazova, M. S., Effect of an electric field on nucleation and growth of crystals. IOP conf. ser., Mater. sci. eng. 2016, 110 (1), 012019.

109. Jiang, S.-D.; Wang, B.-W.; Su, G.; Wang, Z.-M.; Gao, S., A Mononuclear Dysprosium Complex Featuring Single-Molecule-Magnet Behavior. Angew. Chem. Int. Ed. 2010, 49 (41), 7448-7451.

110. Katoh, K.; Asano, R.; Miura, A.; Horii, Y.; Morita, T.; Breedlove, B. K.; Yamashita, M., Effect of $\mathrm{f}-\mathrm{f}$ interactions on quantum tunnelling of the magnetization: mono- and dinuclear Dy(III) phthalocyaninato triple-decker single-molecule magnets with the same octacoordination environment. Dalton Trans. 2014, 43 (21), 7716-25.

111. Horii, Y.; Katoh, K.; Cosquer, G.; Breedlove, B. K.; Yamashita, M., Weak Dy(III)-Dy(III) Interactions in Dy(III)-Phthalocyaninato Multiple-Decker Single-Molecule Magnets Effectively Suppress Magnetic Relaxation. Inorg. Chem. 2016, 55 (22), 11782-11790.

112. Horii, Y.; Kishiue, S.; Damjanovic, M.; Katoh, K.; Breedlove, B. K.; Enders, M.; Yamashita, M., Supramolecular Approach for Enhancing Single-Molecule Magnet Properties of Terbium(III)Phthalocyaninato Double-Decker Complexes with Crown Moieties. Chem. Eur. J. 2018, 24 (17), 43204327.

113. Wernsdorfer, W.; Aliaga-Alcalde, N.; Hendrickson, D. N.; Christou, G., Exchange-biased quantum tunnelling in a supramolecular dimer of single-molecule magnets. Nature 2002, 416 (6879), 4069.

114. Chibotaru, L. U.; Liviu, F., Magnetic anisotropy in the excited states of low symmetry lanthanide complexes. Phys. Chem. Chem. Phys. 2011, 13, 20086-20090.

115. Aquilante, F.; Autschbach, J.; Carlson, R. K.; Chibotaru, L. F.; Delcey, M. G.; De Vico, L.; Fdez. Galván, I.; Ferré, N.; Frutos, L. M.; Gagliardi, L.; Garavelli, M.; Giussani, A.; Hoyer, C. E.; Li Manni, G.; Lischka, H.; Ma, D.; Malmqvist, P. Å.; Müller, T.; Nenov, A.; Olivucci, M.; Pedersen, T. B.; Peng, D.; 
Plasser, F.; Pritchard, B.; Reiher, M.; Rivalta, I.; Schapiro, I.; Segarra-Martí, J.; Stenrup, M.; Truhlar, D. G.; Ungur, L.; Valentini, A.; Vancoillie, S.; Veryazov, V.; Vysotskiy, V. P.; Weingart, O.; Zapata, F.; Lindh, R., Molcas 8: New capabilities for multiconfigurational quantum chemical calculations across the periodic table. J. Comput. Chem. 2016, 37 (5), 506-541.

116. Widmark, P.-O.; Malmqvist, P.-Å.; Roos, B. O., Density matrix averaged atomic natural orbital (ANO) basis sets for correlated molecular wave functions. Theor. Chem. Acc. 1990, 77 (5), 291-306.

117. Roos, B. O.; Lindh, R.; Malmqvist, P.; Veryazov, V.; Widmark, P., Main Group Atoms and Dimers Studied with a New Relativistic ANO Basis Set. J. Phys. Chem. A 2004, 108 (15), 2851-2858.

118. Roos, B. O.; Lindh, R.; Malmqvist, P.-Å.; Veryazov, V.; Widmark, P.-O.; Borin, A. C., New Relativistic Atomic Natural Orbital Basis Sets for Lanthanide Atoms with Applications to the Ce Diatom and $\mathrm{LuF}_{3}$. J. Phys. Chem. A 2008, 112 (45), 11431-11435.

119. Rotter, M., Using McPhase to calculate magnetic phase diagrams of rare earth compounds. $J$. Magn. Magn. Mater. 2004, 272-276, E481-E482.

120. Baldovi, J. J.; Borras-Almenar, J. J.; Clemente-Juan, J. M.; Coronado, E.; Gaita-Arino, A., Modeling the properties of lanthanoid single-ion magnets using an effective point-charge approach. Dalton Trans. 2012, 41 (44), 13705-10.

121. Sakaue, S.; Fuyuhiro, A.; Fukuda, T.; Ishikawa, N., Dinuclear single-molecule magnets with porphyrin-phthalocyanine mixed triple-decker ligand systems giving SAP and SP coordination polyhedra. Chem. Commun. 2012, 48 (43), 5337-5339.

122. Chen, Y.-C.; Liu, J.-L.; Ungur, L.; Liu, J.; Li, Q.-W.; Wang, L.-F.; Ni, Z.-P.; Chibotaru, L. F.; Chen, X.-M.; Tong, M.-L., Symmetry-Supported Magnetic Blocking at $20 \mathrm{~K}$ in Pentagonal Bipyramidal Dy(III) Single-Ion Magnets. J. Am. Chem. Soc. 2016, 138 (8), 2829-2837.

123. Katoh, K.; Kajiwara, T.; Nakano, M.; Nakazawa, Y.; Wernsdorfer, W.; Ishikawa, N.; Breedlove, B. K.; Yamashita, M., Magnetic Relaxation of Single-Molecule Magnets in an External Magnetic Field: An Ising Dimer of a Terbium(III)-Phthalocyaninate Triple-Decker Complex. Chem. Eur. J. 2011, 17 (1), 117122.

124. Liu, J.; Isshiki, H.; Katoh, K.; Morita, T.; Breedlove, B. K.; Yamashita, M.; Komeda, T., First observation of a Kondo resonance for a stable neutral pure organic radical, 1,3,5-triphenyl-6-oxoverdazyl, adsorbed on the $\mathrm{Au}(111)$ surface. J. Am. Chem. Soc. 2013, 135 (2), 651-8.

125. CrysAlisPro Software system, 1.171.39.46; Agilent Technologies UK Ltd, Oxford, UK., 2013.

126. Sheldrick, G., A short history of SHELX. Acta Crystallogr. A 2008, 64 (1), 112-122.

127. Kabuto, C.; Akine, S.; Nemoto, T.; Kwon, E., Release of Software (Yadokari-XG 2009) for Crystal Structure Analyses. Nihon Kessho Gakkaishi 2009, 51 (3), 218-224. 